TRANSACTIONS OF THE

AMERICAN MATHEMATICAL SOCIETY

Volume 365, Number 6, June 2013, Pages 3193-3225

S 0002-9947(2012)05732-1

Article electronically published on November 20, 2012

\title{
KRECK-STOLZ INVARIANTS FOR QUATERNIONIC LINE BUNDLES
}

\author{
DIARMUID CROWLEY AND SEBASTIAN GOETTE
}

\begin{abstract}
We generalise the Kreck-Stolz invariants $s_{2}$ and $s_{3}$ by defining a new invariant, the $t$-invariant, for quaternionic line bundles $E$ over closed spinmanifolds $M$ of dimension $4 k-1$ with $H^{3}(M ; \mathbb{Q})=0$ such that $c_{2}(E) \in H^{4}(M)$ is torsion. The $t$-invariant classifies closed smooth oriented 2 -connected rational homology 7-spheres up to almost-diffeomorphism, that is, diffeomorphism up to a connected sum with an exotic sphere. It also detects exotic homeomorphisms between such manifolds.

The $t$-invariant also gives information about quaternionic line bundles over a fixed manifold, and we use it to give a new proof of a theorem of Feder and Gitler about the values of the second Chern classes of quaternionic line bundles over $\mathbb{H} P^{k}$. The $t$-invariant for $S^{4 k-1}$ is closely related to the Adams $e$-invariant on the $(4 k-5)$-stem.
\end{abstract}

\section{INTRODUCTION}

In 25], Kreck and Stolz introduced invariants $s_{1}, s_{2}, s_{3} \in \mathbb{Q} / \mathbb{Z}$ of certain closed smooth oriented simply connected 7 -manifolds $M$ that completely characterise $M$ up to diffeomorphism. The $s$-invariants are defect invariants based on index theorems for Dirac operators on 8-manifolds: the invariant $s_{1}$, which equals the EellsKuiper invariant $\mu$ of [17] if $M$ is spin, is the defect of the untwisted Dirac operator, whereas $s_{2}$ and $s_{3}$ are the defects of the Dirac operator twisted by certain complex line bundles.

In this paper we define a defect invariant, the $t$-invariant, based on index theorems for Dirac operators twisted by quaternionic line bundles. Let $\operatorname{Bun}(M)$ denote the set of isomorphism classes of quaternionic line bundles over $M$. The second Chern class defines a function

$$
c_{2}: \operatorname{Bun}(M) \longrightarrow H^{4}(M) .
$$

Let $\operatorname{Bun}_{0}(M)$ denote the subset of bundles for which this class is torsion. Now suppose that $M$ is a closed smooth spin $(4 k-1)$-manifold such that the group $H^{3}(M ; \mathbb{Q})$ vanishes. Then the $t$-invariant is a function

$$
t_{M}: \operatorname{Bun}_{0}(M) \longrightarrow \mathbb{Q} / \mathbb{Z}
$$

Received by the editors January 5, 2011 and, in revised form, October 22, 2011 and October 25,2011 .

2010 Mathematics Subject Classification. Primary 58J28, 57R55; Secondary 57R20.

Key words and phrases. $\eta$-invariant, quaternionic line bundle, 7-manifold, smooth structure, Kirby-Siebenmann invariant.

The second author was supported in part by SFB-TR 71 "Geometric Partial Differential Equations". 
see Definition [1.4. It is a precise generalisation of the invariants $s_{2}$ and $s_{3}$ in dimension 7, as we explain in Section 2.c, and falls into Bunke's general framework of [10, where it is discussed in Section 5.5.

The first-named author established a complete classification of 2-connected rational homology 7 -spheres in 12 using a certain extrinsically defined quadratic linking form $q_{M}: H^{4}(M) \rightarrow \mathbb{Q} / \mathbb{Z}$ whose values are calculated using a spin 8-manifold $W$ with boundary $\partial W=M$. Theorem 0.1 below, which is a reformulation of Theorem 2.4, states that the $t$-invariant $t_{M}$ is a refinement of $q_{M}$. Moreover, as a defect invariant, the $t$-invariant has an intrinsic definition, see (1.9), given via $\eta$-invariants arising from the Atiyah-Patodi-Singer index theorem of [5]. Note that for rational homology spheres $M$, we have $\operatorname{Bun}_{0}(M)=\operatorname{Bun}(M)$ because $H^{4}(M)$ is a torsion group.

0.1. Theorem. Let $M$ be a closed smooth 2-connected oriented rational homology 7-sphere. Then

(1) the $\operatorname{map} c_{2}: \operatorname{Bun}(M) \rightarrow H^{4}(M)$ is onto,

(2) for all $E \in \operatorname{Bun}(M), q_{M}\left(c_{2}(E)\right)=12 t_{M}(E)$,

(3) the map $c_{2} \times t_{M}: \operatorname{Bun}(M) \rightarrow H^{4}(M) \times \mathbb{Q} / \mathbb{Z}$ is injective.

We shall say that $M$ and $N$ have isomorphic t-invariants if there is a group isomorphism $A: H^{4}(M) \rightarrow H^{4}(N)$ and a set bijection $B: \operatorname{Bun}_{0}(M) \rightarrow \operatorname{Bun}_{0}(N)$ which are compatible with the second Chern class and the $t$-invariant; see Definition [1.4. Applying [12, Theorem A] we obtain the following expanded version of Corollary 2.5.

0.2. Corollary. Let $N$ and $M$ be closed smooth 2-connected oriented rational homology 7-spheres. Then $N$ is diffeomorphic to $M$ if and only if $M$ and $N$ have isomorphic t-invariants and their Eells-Kuiper invariants agree: $\mu(N)=\mu(M)$.

Our first application of the $t$-invariant was inspired by recent discoveries of Grove, Verdiani and Ziller [21]. In [19] the second-named author used Corollary 0.2 to classify the manifolds $P_{k}$ constructed in [21] by calculating their $t$ - and $\mu$-invariants intrinsically; see Example 2.8 .

A further feature of the $t$-invariant in dimension 7 is that it detects exotic homeomorphisms: these are orientation-preserving homeomorphisms $h: N \cong M$ which are not homotopic to piecewise linear homeomorphisms. As we explain in Section 2.b the results of Kirby and Siebenmann 26] imply that for a homeomorphism $h: N \cong M$ of closed smooth oriented 2-connected rational homology 7-spheres, there is an invariant

$$
\kappa(h) \in H^{3}(M ; \mathbb{Z} / 2),
$$

depending only on the homotopy class of $h$, such that $h$ is exotic if and only if $\kappa(h) \neq$ 0 . Note that $c_{2}$ induces a surjection $\operatorname{Bun}(M) \rightarrow H^{4}(M, \mathbb{Z})$ by Theorem 0.1 (1) above. The following expanded version of Theorem 2.9 shows that the KirbySiebenmann invariant of $h$ can be computed using the induced map $h^{*}: \operatorname{Bun}(M) \rightarrow$ $\operatorname{Bun}(N)$ and the $t$-invariants of $N$ and $M$.

0.3. Theorem. Let $M, N$ be 2-connected oriented rational homology 7-spheres. An orientation-preserving homeomorphism $h: N \rightarrow M$ is exotic if and only if

$$
t_{M} \neq t_{N} \circ h^{*} .
$$


More precisely, for all $E \in \operatorname{Bun}(M)$,

$$
\left(\kappa(h) \smile c_{2}(E)\right)[M]_{2}=t_{N}\left(h^{*} E\right)-t_{M}(E) \in \mathbb{Z} / 2 \subset \mathbb{Q} / \mathbb{Z},
$$

where $[M]_{2}$ generates $H_{7}(M ; \mathbb{Z} / 2)$.

We now change our focus from the base-space manifolds to the bundles themselves. For the simplest manifolds $M=S^{4 k-1}$ we have the $t$-invariant

$$
t_{S^{4 k-1}}: \operatorname{Bun}\left(S^{4 k-1}\right) \cong \pi_{4 k-2}\left(S^{3}\right) \longrightarrow \mathbb{Q} / \mathbb{Z} .
$$

Given its relationship to the Dirac operator and hence the $\widehat{A}$-genus, one might expect that the $t$-invariant is related to the Adam's $e$-invariant $e: \pi_{4 k-5}^{S} \rightarrow \mathbb{Q} / \mathbb{Z}$ of 11. Theorem 1.12, restated immediately below, bears out this expectation.

0.4. Theorem. Assume that $k \geq 3$. For any homotopy sphere $\Sigma^{4 k-1}$ the homomorphism

$$
t_{\Sigma}: \operatorname{Bun}(\Sigma) \cong \pi_{4 k-2}\left(S^{3}\right) \longrightarrow \mathbb{Q} / \mathbb{Z}
$$

may be identified with the composition

$$
-e \circ S: \pi_{4 k-2}\left(S^{3}\right) \longrightarrow \pi_{4 k-5}^{S} \longrightarrow \mathbb{Q} / \mathbb{Z},
$$

where $-e: \pi_{4 k-5}^{S} \rightarrow \mathbb{Q} / \mathbb{Z}$ and $S: \pi_{4 k-2} \rightarrow \pi_{4 k-5}^{S}$ are respectively the negative of the Adams e-invariant, $e_{\mathbb{R}}^{\prime}$ of [1, Proposition 7.14], and the stabilisation homomorphism.

A key feature of quaternionic bundles is that both the quaternions $\mathbb{H}$ and their group of units $S^{3} \subset \mathbb{H}$ are non-abelian. As a result the classifying space $B S^{3}=$ $\mathbb{H} P^{\infty}$ is not an $\mathrm{H}$-space, and for a general space $X, \operatorname{Bun}(X) \equiv\left[X, B S^{3}\right]$ does not have a naturally defined group structure. This leads to the fact that it is often a difficult problem to determine the image of $c_{2}$. For the case $X=\mathbb{H} P^{k}$ and the map

$$
c_{2}: \operatorname{Bun}\left(\mathbb{H} P^{k}\right) \longrightarrow H^{4}\left(\mathbb{H} P^{k}\right)
$$

one says that an integer $c$ is $k$-realisable if $c \cdot c_{2}(H) \in \operatorname{Im}\left(c_{2}\right)$, where $H$ is the tautological bundle on $\mathbb{H} P^{k}$ and $c_{2}(H)$ is the universal second Chern class. In Theorem 3.1, we state the value of $t$-invariants of quaternionic line bundles on $S^{4 k-1}$ that are pulled back from $\mathbb{H} P^{k-1}$ by the Hopf fibration. This leads to a new proof of the Feder-Gitler criteria [18, Theorem 1.1] for $k$-realizability.

The rest of the paper is organised as follows: in Section 1 we define the $t$-invariant from both the intrinsic analytic point of view and the extrinsic topological point of view. This section also introduces the basic concepts and tools required for our analysis of quaternionic line bundles. In Section 2 we consider the $t$-invariant for 7-manifolds. We update the classification results of 12 in Theorem 2.4 and we show that the $t$-invariant detects exotic homeomorphisms in Theorem [2.9, In Section $2 . c$ we show how the $t$-invariant on simply connected 7 -manifolds relates to the $s$-invariants and their generalisations by Hepworth in 23]. Section 3 gives our proof of the Feder-Gitler criteria as well as several explicit computations of the $t$-invariant for certain bundles over $S^{7}, S^{11}$ and $S^{15}$.

\section{SECONDARY INVARIANTS}

In this section we define the $t$-invariant which is an invariant of pairs $(M, E)$, where $M$ is a closed spin $(4 k-1)$-manifold and $E$ is a quaternionic line bundle over $M$. We will give both an extrinsic and an intrinsic definition of the $t$-invariant. Whereas the extrinsic definition using zero bordisms is easier to handle in many cases, the intrinsic definition using $\eta$-invariants is finer for manifolds that are only 
rationally zero bordant and may sometimes be computed when 0-bordisms cannot be found. We will state and prove properties of the $t$-invariant in both settings when we are able to because the extrinsic proofs are easier in most cases.

The section is organised as follows. Section 1.a briefly reviews quaternionic line bundles. Section 1.b defines the $t$-invariant and identifies some of its basic properties. Section 1.c introduces the notion of a quaternionic divisor used in the proof of Theorem 1.12, which occupies most of Section 1.d.

1.a. Quaternionic line bundles. Let $\mathbb{H}, \mathbb{H}^{\times}$and $S^{3}$ denote respectively the quaternions, the non-zero quaternions and the unit quaternions. A quaternionic line bundle, $E \rightarrow X$ or simply $E$, over a space $X$ is a complex rank 2 vector bundle $V \rightarrow X$ together with a reduction of its structure group to $\mathbb{H}^{\times}$. An isomorphism of quaternionic line bundles is a vector bundle isomorphism respecting the quaternionic structures. As an example, consider the tautological bundle

$$
H \longrightarrow \mathbb{H} P^{\infty}=B S^{3} \text {. }
$$

Since the structure group $\mathbb{H}^{\times}$of a quaternionic line bundle can always be reduced to $S^{3}$, and this reduction is unique up to a contractible choice, quaternionic line bundles are classified by the homotopy classes of maps to $B S^{3}$. So

$$
\operatorname{Bun}(X)=\left[X, B S^{3}\right]
$$

describes the set of isomorphism classes of quaternionic line bundles over a space $X$. By abuse of notation, we will sometimes write $E \in \operatorname{Bun}(X)$ to say that $E$ is a quaternionic line bundle over $X$.

The integral cohomology of $B S^{3}$ is generated by the universal second Chern class $c_{2}(H) \in H^{4}\left(\mathbb{H} P^{\infty}\right)$. So to each bundle $E \rightarrow X$ with classifying map $f: X \rightarrow$ $B S^{3}$ we may associate the characteristic class

$$
c_{2}(E):=f^{*} c_{2}(H) \in H^{4}(X) .
$$

In this way we obtain a map

$$
c_{2}: \operatorname{Bun}(X) \longrightarrow H^{4}(X), \quad E \longmapsto c_{2}(E) .
$$

We will also consider the subset $\operatorname{Bun}_{0}(X)$ of quaternionic line bundles $E$ where $c_{2}(E)$ is a torsion, that is, with $c_{2}(E)=0 \in H^{4}(X ; \mathbb{Q})$.

Note that the theory of quaternionic bundles is not quite as straightforward as in the complex case. First, the tensor product of two quaternionic line bundles is merely a spin four-dimensional real vector bundle. In fact, every four-dimensional spin vector bundle arises this way. In particular, there is no canonical group structure on quaternionic line bundles.

Next, quaternionic line bundles are not classified by their second Chern class. The reason is that $B S^{3}$ is not an Eilenberg-MacLane space. For example, the quaternionic line bundles on $S^{7}$ are classified by

$$
\pi_{7}\left(B S^{3}\right) \cong \pi_{6}\left(S^{3}\right) \cong \mathbb{Z} / 12,
$$

but of course $H^{4}\left(S^{7}\right)=0$. On the other hand, for a general space $X$, not all the elements in $H^{4}(X)$ are realised as $c_{2}(E)$ for a quaternionic line bundle $E \rightarrow X$ : for example, as is well known and explained in Section 3 , if $X=\mathbb{H} P^{2}$, then the image of $c_{2}$ in the group $H^{4}\left(\mathbb{H} P^{2}\right) \cong \mathbb{Z}$ is precisely all those classes $c \cdot c_{2}(H)$ such that $c(c-1) / 2$ is congruent to $0 \bmod 12$. 
1.b. Modified Kreck-Stolz invariants. Let $M$ be a closed spin manifold of dimension $4 k-1$ and let $E \rightarrow M$ be a complex vector bundle over $M$. As observed by Kreck and Stolz, a source of $\mathbb{Q} / \mathbb{Z}$-valued invariants of the pair $(M, E)$ is the following index theorem.

1.1. Theorem (Atiyah-Singer [6, III, Theorem 5.3]). Let $X^{4 k}$ be a closed, smooth spin manifold and let $E$ be a complex vector bundle over $X$. Then the index of $D_{X}^{E}$, the Dirac operator of $X$ twisted by $E$, can be computed by the following equation:

$$
\operatorname{ind}\left(D_{X}^{E}\right)=(\widehat{A}(T X) \operatorname{ch}(E))[X] \in \mathbb{Z},
$$

where $\widehat{A}(T X)$ and $\operatorname{ch}(E) \in H^{*}(X)$ denote respectively the $\widehat{A}$-genus of $T X$ and the Chern character of $E$ and $[X] \in H_{4 k}(X)$ is the fundamental class of $X$.

Assume that $(M, E)$ as above bounds a spin manifold and complex vector bundle $(W, \bar{E})$ and that the characteristic class $\widehat{A}(T W) \operatorname{ch}(\bar{E})$ has a natural interpretation in $H^{4 k}(W, M ; \mathbb{Q})$. Then evaluation on the relative fundamental cycle $[W, M]$ gives a rational number which, by Theorem [1.1] is an invariant of $(M, E) \bmod \mathbb{Z}$. The Atiyah-Patodi-Singer index theorem 1.2 below can also be used to identify this invariant as an intrinsic invariant of $(M, E)$. In the remainder of this section, we carry out this program for quaternionic line bundles.

Recall that a real structure $s$ on a complex vector bundle $E \rightarrow X$ is an antilinear automorphism with $s^{2}=1$. Similarly, a quaternionic structure $j$ on $E$ is an anti-linear automorphism with $j^{2}=-1$. A quaternionic vector bundle $E \rightarrow X$ can be regarded as a complex vector bundle with complex structure $i$ and quaternionic structure $j$. We can always choose a quaternionic-Hermitian metric on $E$ and a connection such that the quaternionic structure is parallel.

If $X^{4 k}$ is a spin manifold and $k$ is even, then the complex spinor bundle $\Sigma X$ of $X$ carries a real structure $s$ that is parallel with respect to the Levi-Civita connection. Then $s \otimes j$ is a quaternionic structure on $\Sigma X \otimes_{\mathbb{C}} E$ that commutes with the Dirac operator. This implies that $\mathbb{H}$ acts on all eigenspaces of $D_{X}^{E}$, in particular

$$
\operatorname{ind}\left(D_{X}^{E}\right) \in 2 \mathbb{Z} \quad \text { if } \operatorname{dim} X \equiv 0 \bmod 8 .
$$

Because $c_{2}$ generates the ring of characteristic classes of quaternionic line bundles, there exists a unique universal characteristic class $\mathrm{ch}^{\prime}$ of quaternionic line bundles such that

$$
2-\operatorname{ch}(E)=c_{2}(E) \operatorname{ch}^{\prime}(E) .
$$

In fact, one computes that

$$
\operatorname{ch}^{\prime}(E)=1-\frac{1}{12} c_{2}(E)+\ldots .
$$

Now suppose that $M$ bounds a compact spin manifold $W$ such that $E$ is the restriction of a quaternionic line bundle $\bar{E} \rightarrow W$. In other words,

$$
[M, E]=0 \in \Omega_{*}^{\mathrm{Spin}}\left(B S^{3}\right) .
$$

Assume moreover that $H^{3}(M ; \mathbb{Q})=0$ and that $E \in \operatorname{Bun}_{0}(M)$. Then $c_{2}(E)=0 \in$ $H^{4}(M ; \mathbb{Q})$, and because of the exact sequence

$$
H^{3}(M ; \mathbb{Q}) \longrightarrow H^{4}(W, M ; \mathbb{Q}) \longrightarrow H^{4}(W ; \mathbb{Q}) \longrightarrow H^{4}(M ; \mathbb{Q}),
$$

the class $c_{2}(\bar{E}) \in H^{4}(W ; \mathbb{Z})$ has a unique lift $\bar{c}_{2}(\bar{E}) \in H^{4}(W, M ; \mathbb{Q})$. 
As a preliminary definition of the $t$-invariant, let us consider the quantity

$$
\tau_{M}(E):=-\frac{1}{a_{k+1}}\left(\widehat{A}(T W) \operatorname{ch}^{\prime}(\bar{E}) \bar{c}_{2}(\bar{E})\right)[W, M] \in \mathbb{Q} / \mathbb{Z},
$$

where $a_{j}$ is 1 if $j$ is even and 2 if $j$ is odd. Given another compact spin manifold $W^{\prime}$ with $\partial W^{\prime}=M$ and a quaternionic line bundle $\bar{E}^{\prime} \rightarrow W^{\prime}$ extending $W$, we may consider

$$
F=\bar{E}^{\prime} \cup_{E} \bar{E} \longrightarrow X=W^{\prime} \cup_{M}(-W),
$$

where $-W$ carries the reversed orientation. Theorem 1.1 and property (1.2) above imply that the two right-hand sides of (1.6) differ by

$$
\frac{1}{a_{k+1}}(\widehat{A}(T X)(\operatorname{ch}(F)-2))[X]=\frac{1}{a_{k+1}}\left(\operatorname{ind}\left(D_{X}^{F}\right)-2 \operatorname{ind}\left(D_{X}\right)\right) \in \mathbb{Z},
$$

so that $\tau_{M}(E)$ is independent of the choice of the pair $(W, \bar{E})$.

We now want to define $\tau_{M}(E)$ intrinsically on $M$, so that we can drop condition (1.5). We equip $T M$ with a Riemannian metric $g$. It gives rise to a Levi-Civita connection $\nabla^{T M}$ on $T M$. Let $D$ be the untwisted spin Dirac operator of $(M, g)$ acting on the complex spinor bundle $\Sigma M \rightarrow M$. We also equip the quaternionic line bundle $E$ with a quaternionic Hermitian metric $h^{E}$ and a compatible connection $\nabla^{E}$. As above, $D_{M}^{E}$ denotes the spin Dirac operator twisted by $\left(E, \nabla^{E}, h^{E}\right)$. Let $\eta\left(D_{M}\right)$ and $\eta\left(D_{M}^{E}\right)$ denote the Atiyah-Patodi-Singer $\eta$-invariants and put $h\left(D_{M}\right)=\operatorname{dim} \operatorname{ker} D_{M}$ and $h\left(D_{M}^{E}\right)=\operatorname{dim} \operatorname{ker} D_{M}^{E}$.

Let $\left(\Omega^{\bullet}(M), d\right)$ denote the de Rham complex of $M$. We consider the characteristic Chern-Weil forms

$$
\widehat{A}\left(T M, \nabla^{T M}\right), \quad c_{2}\left(E, \nabla^{E}\right), \quad \text { and } \quad \operatorname{ch}\left(E, \nabla^{E}\right) \in \operatorname{ker} d \subset \Omega^{\bullet}(M)
$$

that represent the corresponding characteristic classes in de Rham cohomology. The ring of Ad-invariant polynomials on $\mathfrak{s p}(1) \cong \mathfrak{s u}(2) \subset \mathfrak{g l}(2, \mathbb{C})$ is freely generated by the complex determinant $\sigma_{2}=\operatorname{det}_{\mathbb{C}}$ that gives rise to the second ChernWeil Chern class $c_{2}$. As in (1.3), there is then a unique characteristic Chern-Weil form $\operatorname{ch}^{\prime}\left(E, \nabla^{E}\right)$ such that

$$
2-\operatorname{ch}\left(E, \nabla^{E}\right)=c_{2}\left(E, \nabla^{E}\right) \operatorname{ch}^{\prime}\left(E, \nabla^{E}\right) .
$$

If $c_{2}(E) \in H^{4}(M)$ is torsion, then we can find $\hat{c}_{2}\left(E, \nabla^{E}\right) \in \Omega^{3}(M)$ such that

$$
d \hat{c}_{2}\left(E, \nabla^{E}\right)=c_{2}\left(E, \nabla^{E}\right) \text {. }
$$

If $H^{3}(M ; \mathbb{Q})=0$, the de Rham cohomology of $M$ vanishes in this degree, so the form $\hat{c}_{2}\left(E, \nabla^{E}\right)$ is uniquely determined modulo exact forms.

We consider the expression

$$
\begin{aligned}
t_{M}(E):=\frac{1}{a_{k+1}}\left(\frac{\eta+h}{2}\left(D_{M}^{E}\right)\right. & -(\eta+h)\left(D_{M}\right) \\
& \left.+\int_{M} \widehat{A}\left(T M, \nabla^{T M}\right)\left(\hat{c}_{2} \operatorname{ch}^{\prime}\right)\left(E, \nabla^{E}\right)\right) \in \mathbb{R} / \mathbb{Z} .
\end{aligned}
$$

We will see below that $t_{M}(E)$ is indeed well-defined.

Now assume that $(M, g)$ bounds a Riemannian spin manifold $(W, \bar{g})$ and that the triple $\left(E, \nabla^{E}, h^{E}\right)$ extends to $\left(\bar{E}, \nabla^{\bar{E}}, h^{\bar{E}}\right) \rightarrow W$. Then the pair

$$
\bar{c}_{2}\left(\bar{E}, \nabla^{\bar{E}}\right)=\left(c_{2}\left(\bar{E}, \nabla^{\bar{E}}\right), \hat{c}_{2}\left(E, \nabla^{E}\right)\right) \in \Omega^{4}(W) \oplus \Omega^{3}(M)
$$


is closed in the relative de Rham complex. It represents a lift $\bar{c}_{2}(\bar{E})$ of $c_{2}(\bar{E})$ to $H^{4}(W, M ; \mathbb{R})$. By the variation formulas for Chern-Simons classes, $\bar{c}_{2}(\bar{E})$ does not depend on $\nabla^{E}$ and $h^{E}$. Note that closed forms on $W$ act on pairs as above by multiplication in both factors, and that evaluation on the relative fundamental cycle $[W, M]$ is given by

$$
(\alpha, \beta)[W, M]=\int_{W} \alpha-\int_{M} \beta .
$$

Let us assume that $(W, \bar{g})$ and $\left(\bar{E}, \nabla^{\bar{E}}, h^{\bar{E}}\right)$ are of product type near the boundary. In this case, the Atiyah-Patodi-Singer boundary conditions for the spin Dirac operator $D_{W}^{\bar{E}}$ twisted by $\left(\bar{E}, \nabla^{\bar{E}}, h^{\bar{E}}\right)$ are defined.

1.2. Theorem (Atiyah-Patodi-Singer [5, I, (4.3)]). Let $(M, g)=\partial(W, \bar{g})$ as above and let $\left(\bar{E}, \nabla^{\bar{E}}, h^{\bar{E}}\right)$ denote a complex vector bundle with Hermitian connection and parallel metric over $W$. Then

$$
\operatorname{ind}\left(D_{W}^{\bar{E}}\right)=\int_{W} \widehat{A}\left(W, \nabla^{W}\right) \operatorname{ch}\left(\bar{E}, \nabla^{\bar{E}}\right)-\frac{\eta+h}{2}\left(D_{M}^{E}\right) \in \mathbb{Z} .
$$

Again, if the Dirac operator commutes with a quaternionic structure as in (1.2), then the index above is even.

1.3. Proposition. Let $\left(E, \nabla^{E}, h^{E}\right)$ be a quaternionic line bundle with quaternionic connection and parallel quaternionic-Hermitian metric over $(M, g)$. Assume that $H^{3}(M ; \mathbb{Q})=0$ and that $c_{2}(E)$ is a torsion class.

(1) If $(M, E)$ bounds $(W, \bar{E})$ as above, then

$$
\tau_{M}(E)=t_{M}(E) \text {. }
$$

(2) The invariant $t_{M}(E)$ is always rational.

Proof. For (11), we use the Atiyah-Patodi-Singer index theorem as in [16] and 25] together with (1.2), (1.4), (1.10) and (1.11). We find that

$$
\begin{aligned}
-\left(\widehat{A}(T W) \bar{c}_{2}(\bar{E}) \operatorname{ch}^{\prime}(\bar{E})\right)[W, M] & \\
=- & \int_{W} \widehat{A}\left(T W, \nabla^{T W}\right)(2-\mathrm{ch})\left(\bar{E}, \nabla^{\bar{E}}\right) \\
& \quad+\int_{M} \widehat{A}\left(T M, \nabla^{T M}\right)\left(\hat{c}_{2} \mathrm{ch}^{\prime}\right)\left(E, \nabla^{E}\right) \\
\equiv & \frac{\eta+h}{2}\left(D_{M}^{E}\right)-(\eta+h)\left(D_{M}\right) \\
& \quad+\int_{M} \widehat{A}\left(T M, \nabla^{T M}\right)\left(\hat{c}_{2} \operatorname{ch}^{\prime}\right)\left(E, \nabla^{E}\right) \quad \bmod a_{k+1} \mathbb{Z} .
\end{aligned}
$$

For (2), we use that the Spin bordism group $\Omega_{4 j-1}^{\text {Spin }}$ is a finite $\mathbb{Z} / 2$-vector space for $j>0$ by [2]. Since $H^{*}\left(B S^{3} ; \mathbb{Z}\right)=\mathbb{Z}\left[c_{2}\right]$, where $c_{2}$ has degree 4 , we see that the $(4 k-1)$-line of the $E_{2}$-page of the the Atiyah-Hirzebruch spectral sequence computing $\Omega_{4 k-1}^{\text {Spin }}\left(B S^{3}\right)$ consists of summands $E_{4 j, 4(k-j)-1}=H^{4 j}\left(\mathbb{H} P^{\infty} ; \Omega_{4(k-j)-1}^{\text {Spin }}\right)$. For any spin-manifold $X$ of dimension $4(k-j)-1$ we have the manifold $X \times \mathbb{H} P^{k}$ which is again spin since $\mathbb{H} P^{k}$ is a spin-manifold. Moreover we have the obvious map $X \times \mathbb{H} P^{k} \rightarrow \mathbb{H} P^{k} \rightarrow \mathbb{H} P^{\infty}$ and thus we see that each element of $E_{4 j, 4(k-j)-1}$ is represented by a permanent cycle of order 2 . It follows that $\Omega_{4 k-1}^{\mathrm{Spin}}\left(\mathbb{H} P^{\infty}\right)$ is 
a finite $\mathbb{Z} / 2$-vector space. Hence for any $S^{3}$-bundle $(M, E)$, there is $n \in\{1,2\}$ such that $(M, E)^{\sqcup n}$ (or equivalently $(M, E)^{\sharp n}$ ) bounds a spin manifold $W$ with a quaternionic line bundle $\bar{E}$ that extends $E^{\sqcup n}$. By (1),

$$
n t_{M}(E)=\tau_{M} \sqcup n\left(E^{\sqcup n}\right) \in \mathbb{Q} / \mathbb{Z} .
$$

The argument above also shows that, in general, the extrinsic definition (1.6) gives the value of $t_{M}(E)$ only up to multiples of $\frac{1}{n}$ in $\mathbb{Q} / \mathbb{Z}$.

A similar argument as above shows that $t_{M}(E)$ depends neither on the choice of the metric $g$ on $M$ nor on the connection $\nabla^{E}$ with the parallel metric $h^{E}$ on $E$. To see this, regard two sets of these geometric data on $M$ and $E$. Because $\partial(M \times$ $[0,1])=M \sqcup(-M)$, we can extend these data to $W=M \times[0,1]$. Then $\tau_{M \sqcup(-M)}(E \sqcup$ $E)=0$, and Proposition 1.3 shows that $t_{M}(E)$ is well-defined.

We finally give the definition of the $t$-invariant.

1.4. Definition. Let $M$ be a closed smooth spin $(4 k-1)$-manifold with $H^{3}(M ; \mathbb{Q})=$ 0 . Then we use equation (1.9) above to define the $t$-invariant of $M$ as the function

$$
t_{M}: \operatorname{Bun}_{0}(M) \longrightarrow \mathbb{Q} / \mathbb{Z}, \quad E \longmapsto t_{M}(E) .
$$

We shall say that $M$ and $N$ have isomorphic t-invariants if there is a group isomorphism $A: H^{4}(M) \rightarrow H^{4}(N)$ and a set bijection $B: \operatorname{Bun}_{0}(M) \rightarrow \operatorname{Bun}_{0}(N)$ which are compatible with the second Chern class and the $t$-invariant; i.e.,

$$
\forall E \in \operatorname{Bun}_{0}(M), \quad c_{2}(B E)=A c_{2}(E) \quad \text { and } \quad t_{N}(B E)=t_{M}(E) .
$$

We conclude this subsection by recording some basic facts about the $t$-invariant.

1.5. Proposition. The function $t_{M}: \operatorname{Bun}_{0}(M) \rightarrow \mathbb{Q} / \mathbb{Z}$ has the following properties:

(1) Triviality: for the trivial bundle $\varepsilon:=M \times \mathbb{H}$, we have $t_{M}(\varepsilon)=0$.

(2) Additivity with respect to connected sum of bundles and manifolds:

$$
t_{M_{0} \sharp M_{1}}\left(E_{0} \sharp E_{1}\right)=t_{M_{0}}\left(E_{0}\right)+t_{M_{1}}\left(E_{1}\right) \in \mathbb{Q} / \mathbb{Z} \text {. }
$$

(3) Naturality under almost-diffeomorphisms: if $\Sigma$ is a homotopy sphere and there is a spin diffeomorphism $f: N \sharp \Sigma \cong M$, then

$$
t_{N} \circ f^{*}=t_{M},
$$

where we identify $N$ and $N \sharp \Sigma$ as spaces so that $\operatorname{Bun}_{0}(N \sharp \Sigma)=\operatorname{Bun}_{0}(N)$.

1.6. Remark. (1) Properties (10)-(3) hold for any generalised Kreck-Stolz invariants as we now explain. Let $M$ be a closed odd-dimensional manifold, equipped with a geometric Dirac operator $D$, and let $P$ denote the corresponding local index class. For the $t$-invariant, we take the spin Dirac operator and the $\widehat{A}$-class, but one could also consider the signature operator and the $L$-class or something similar. Then let $E \rightarrow M$ be a vector bundle of a specific type, and let $D^{E}$ denote the operator $D$ twisted by $E$. If there exists a natural class $\alpha \in \Omega^{\bullet}(M) /$ im $d$ such that $d \alpha=\operatorname{ch}(E)-$ rk $E$, then one can consider the invariant

$$
\frac{\eta+h}{2}\left(D^{E}\right)-\frac{\eta+h}{2}(D) \operatorname{rk}(E)-\int_{M} P\left(T M, \nabla^{T M}\right) \alpha \in \mathbb{R} / a \mathbb{Z},
$$

where $a \in \mathbb{Z}$ depends on the index problem. Note that the Atiyah-PatodiSinger $\rho$ - and $\xi$-invariants [5, II] are special cases, where $\left(E, \nabla^{E}\right)$ is flat, so 
one can take $\alpha=0$. The proof below will work equally well for all invariants of this type.

(2) We shall see later in Theorem 2.9 that the $t$-invariant is not in general preserved by homeomorphisms.

Proof of Proposition 1.5. For the trivial bundle $\varepsilon$, one can choose $\hat{c}_{2}=0$, so (1.9) gives $t_{M}(\varepsilon)=0$, and (11) follows.

For (2), we choose points $p_{i} \in M_{i}$ and assume that $\left(E_{i}, \nabla^{E_{i}}\right)$ is isomorphic to a trivial bundle over a small disk $U_{i}$ around $p_{i}$ in $M_{i}$. Let

$$
W=\left(M_{0} \times[0,1]\right) \natural\left(M_{1} \times[0,1]\right)
$$

denote the boundary connected sum taken at the points $\left(p_{0}, 1\right)$ and $\left(p_{1}, 1\right)$. Put

$$
M=\partial W=\left(M_{0} \sharp M_{1}\right) \sqcup\left(-M_{0}\right) \sqcup\left(-M_{1}\right) .
$$

The boundary connected sum of the pullbacks of $E_{i}$ with respect to the given trivialisations gives a bundle $\bar{E} \rightarrow W$. The pullback connections are trivial on $U_{i} \times$ $[0,1]$, so we get a connection $\nabla^{\bar{E}}$ by gluing.

We note that $d \hat{c}^{2}\left(E_{i}, \nabla^{E_{i}}\right)=c^{2}\left(E_{i}, \nabla^{E_{i}}\right)$ vanishes on $U_{i}$ by assumption. Since $U_{i}$ is contractible, there are representatives $\hat{c}_{2}\left(E_{i}, \nabla^{E_{i}}\right) \in \Omega^{3}\left(M_{i}\right)$ that vanish on $U_{0}$ and $U_{1}$ as well. By pullback and gluing, we obtain a global form $\hat{c}_{2}\left(\bar{E}, \nabla^{\bar{E}}\right) \in$ $\Omega^{3}(W)$ with

$$
d \hat{c}_{2}\left(\bar{E}, \nabla^{\bar{E}}\right)=c_{2}\left(\bar{E}, \nabla^{\bar{E}}\right) .
$$

In particular, the lift $\bar{c}_{2}\left(\bar{E}, \nabla^{\bar{E}}\right)$ is exact in the mapping cone of the pullback homomorphism $\Omega^{\bullet}(W) \rightarrow \Omega^{\bullet}(M)$.

From the extrinsic description (1.6) and Stokes' theorem, we obtain

$$
\begin{aligned}
t_{M_{0} \sharp M_{1}}\left(E_{0} \sharp E_{1}\right)- & t_{M_{0}}\left(E_{0}\right)-t_{M_{1}}\left(E_{1}\right)=\tau_{M}(E) \\
=-\int_{W} \widehat{A}(T W, & \left.\nabla^{T W}\right)\left(d \hat{c}_{2} \mathrm{ch}^{\prime}\right)\left(\bar{E}, \nabla^{\bar{E}}\right) \\
& \quad+\int_{\partial W} \widehat{A}\left(T M, \nabla^{T M}\right)\left(\hat{c}_{2} \mathrm{ch}^{\prime}\right)\left(E, \nabla^{E}\right)=0 .
\end{aligned}
$$

Naturality in (3) is clear for spin diffeomorphisms and follows from additivity for almost-diffeomorphisms: add the trivial bundle $(\Sigma, \varepsilon)$ to $(M, E)$.

1.7. Remark. DeLoup and Massuyeau [15, Definition 2.2] define a quadratic form

$$
\phi_{M, \sigma}: H_{2}(M ; \mathbb{Q} / \mathbb{Z}) \longrightarrow \mathbb{Q} / \mathbb{Z}
$$

for oriented closed 3-manifolds $M$ with a $\operatorname{spin}^{c}$-structure $\sigma$. For the definition, of which the $t$-invariant is an analogue, they use a handlebody $W$ with $\partial W=M$. The Poincaré dual $H^{1}(M ; \mathbb{Q} / \mathbb{Z})$ of $H_{2}(M ; \mathbb{Q} / \mathbb{Z})$ classifies flat complex line bundles over $M$ with finite holonomy. Using the Atiyah-Patodi-Singer index theorem, it is not hard to show that $\phi_{M, \sigma}$ has an intrinsic description in terms of the $\xi$-invariant for flat bundles of [5, II, section 3].

1.c. Divisors. In this subsection we develop the concept of the divisor $(Y, \nu)$ of a quaternionic line bundle $E$ in analogy with divisors of complex line bundles. The main result is Proposition 1.10, which explains how the $\eta$-invariants involved in the intrinsic definition of $t_{M}$ localise near a divisor.

Complex line bundles on algebraic varieties are characterised by divisors, that is, by subvarieties of codimension 1. A similar construction still works for smooth 
manifolds. Let $L \rightarrow M$ be a complex line bundle, and let $s \in \Gamma(L)$ be a section that intersects the zero section $M \subset L$ transversally. Let $\nu \rightarrow Y$ be the normal bundle of the zero set $Y=s^{-1}(0)$ of $s$. Then $\nu$ inherits a complex structure, which is equivalent to the choice of an orientation. Thus a divisor for a complex line bundle is a closed smooth submanifold of codimension 2 with a normal orientation. Two divisors give isomorphic smooth complex line bundles if and only if they represent the same class in $H^{2}(M)$.

If $E \rightarrow M$ is a quaternionic line bundle, we choose a transversal section $s \in \Gamma(E)$ as above and put $Y=s^{-1}(0)$. Then $\left.d s\right|_{Y}:\left.\nu \rightarrow E\right|_{Y}$ is an isomorphism of real vector bundles, so $\nu$ inherits a quaternionic structure. In particular, the normal bundle is oriented, and hence defines a class $c_{2}(E) \in H^{4}(M)$.

1.8. Definition. Let $M$ be a compact smooth manifold. A quaternionic divisor in $M$ is a compact codimension-4 submanifold $Y \subset M$ together with a quaternionic structure on its normal bundle. We assume that $Y$ meets $\partial M$ transversally in $\partial Y$. Two quaternionic divisors in $M \times\{0\}, M \times\{1\}$ are equivalent if they extend to a quaternionic divisor in $M \times[0,1]$.

Note that $\partial Y \subset \partial M$, in particular $\partial Y=\emptyset$ if $M$ is closed. For the equivalence of quaternionic divisors on a compact manifold with boundary, we regard $M \times[0,1]$ as a manifold with boundary

$$
\partial(M \times[0,1])=(-M \times\{0\}) \cup(\partial M \times[0,1]) \cup(M \times\{1\})
$$

after rounding off the corners.

For example, $\mathbb{H} P^{k} \subset \mathbb{H} P^{k+1}$ with the obvious quaternionic structure on its normal bundle is a quaternionic divisor for the tautological bundle $H \rightarrow \mathbb{H} P^{k+1}$. To reconstruct $E \rightarrow M$ from a quaternionic divisor $(Y, \nu)$, let $\nu \rightarrow Y$ be classified by $\xi: Y \rightarrow B S^{3}$. Because $M$ is compact, $\xi$ factors through some $\mathbb{H} P^{k} \subset$ $\mathbb{H} P^{\infty}=B S^{3}$. The Thom space of the tautological bundle over $\mathbb{H} P^{k}$ can be identified with $\mathbb{H} P^{k+1}$. Hence, the Pontryagin-Thom construction gives a classifying map for $E$ as in the following diagram:

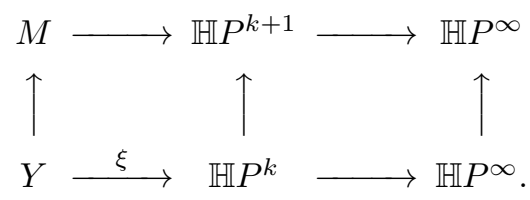

The vertical arrow on the right is given by inserting 0 as the first coordinate and shifting all other coordinates one place to the right. One similarly checks that two quaternionic divisors in $M$ give rise to isomorphic quaternionic line bundles if and only if they are equivalent.

Representing a quaternionic line bundle $E \rightarrow M$ by a quaternionic divisor $(Y, \nu)$ in $M$ allows us to replace the $\eta$-invariants in (1.9) by the $\eta$-invariant of an untwisted Dirac operator on $Y$. However, we will see in Remark 1.15 below that it is not possible to express $t_{M}(E)$ solely in terms of $(Y, \nu)$ without referring to the ambient manifold $M$.

For motivation of Proposition [1.10, we assume first that $(M, E)$ bounds. Hence let $\bar{E} \rightarrow W$ be an extension of $E \rightarrow M$, and let $s \in \Gamma(\bar{E})$ be a transversal section 
of $\bar{E}$ such that $\left.s\right|_{M} \in \Gamma(E)$ is transversal as well. Then we obtain a divisor

$$
(X, \nu):=\left(s^{-1}(0),\left.\bar{E}\right|_{s^{-1}(0)}\right)
$$

of $\bar{E}$ such that $Y:=X \cap M$ is a divisor for $M$.

1.9. Remark. Let $\varepsilon \rightarrow W$ again denote the trivial quaternionic line bundle. Multiplying the section $s$ with scalars from $\varepsilon$, we obtain a bundle morphism $s: \varepsilon \rightarrow \bar{E}$ that is invertible outside $X$. Hence $\varepsilon-\bar{E}$ represents a class in the relative $K$ group $K^{0}(W, W \backslash X)$. Let $s^{*}$ denote the adjoint of $s$.

Along $X$, we still consider the isomorphism $d s:\left.\nu \rightarrow \bar{E}_{\mathbb{R}}\right|_{X}$ and assume that it is an isometry. Multiplication with scalars again gives maps $d s:\left.\left.\nu \otimes \varepsilon\right|_{X} \rightarrow \bar{E}\right|_{X}$ and $d s^{*}:\left.\left.\nu \otimes \bar{E}\right|_{X} \rightarrow \varepsilon\right|_{X}$. The action of $\nu$ on $\varepsilon \oplus \bar{E}$ by $i\left(d s+d s^{*}\right)$ satisfies the axioms of a Clifford multiplication.

This implies that $\varepsilon-\bar{E}$ is a $K$-theoretic direct image in the sense of [4, section 4$]$ and [8, (1.10)] of the trivial one-dimensional complex vector bundle on $X$ under the inclusion $X \hookrightarrow W$. Using the definition of $\hat{A}$ and the formula for the Chern character of the spinor bundle in [4, section 4], it is not hard to check that

$$
\widehat{A}\left(\left.\bar{E}_{\mathbb{R}}\right|_{X},\left.\nabla^{E}\right|_{X}\right) \operatorname{ch}^{\prime}\left(\left.\bar{E}\right|_{X},\left.\nabla^{E}\right|_{X}\right)=1 \in \Omega^{\bullet}(X) .
$$

The bundles $\pi: \bar{E} \rightarrow M$ and $\nu \rightarrow X$ are naturally oriented as real vector bundles by their quaternionic structures. Let $\Omega_{(1)}^{\bullet}$ denote the completion of the smooth forms with compact support with respect to the $L^{1}$-norm. Because $\int \alpha \wedge \beta$ exists for all $\alpha \in \Omega_{(1)}^{\bullet}$ and all forms $\beta \in \Omega^{\bullet}$ with compact support, we will regard elements of $\Omega_{(1)}^{\bullet}$ as form-valued currents. Let $\psi\left(\bar{E}, \nabla^{\bar{E}}\right) \in \Omega_{(1)}^{3}(\bar{E})$ denote the Mathai-Quillen current described in [7, section III.d)] with

$$
d \psi\left(\bar{E}, \nabla^{\bar{E}}\right)=\pi^{*} c_{2}\left(\bar{E}, \nabla^{\bar{E}}\right)-\delta_{0} \in \Omega_{(1)}^{4}(\bar{E}),
$$

where $\delta_{0}$ denotes the distribution of integration over the zero section and the exterior differential $d$ is understood in the weak sense. Because $s$ is a transversal section, the pullback current $s^{*} \psi\left(\bar{E}, \nabla^{\bar{E}}\right) \in \Omega_{(1)}^{3}(W)$ exists and satisfies

$$
d\left(s^{*} \psi\left(\bar{E}, \nabla^{\bar{E}}\right)\right)=c_{2}\left(\bar{E}, \nabla^{\bar{E}}\right)-\delta_{X} \in \Omega_{(1)}^{4}(W)
$$

by [7. (3.37)], where $\delta_{X}$ now denotes the current of integration over $X$. Analogous statements hold for the restriction to $M=\partial W$. We assume again that $H^{3}(M ; \mathbb{Q})=$ 0 and $E \in \operatorname{Bun}_{0}(M)$, so $\hat{c}_{2}\left(E, \nabla^{E}\right)$ is well-defined, and conclude that

$$
d\left(\left(\hat{c}_{2}-s^{*} \psi\right)\left(E, \nabla^{E}\right)\right)=\delta_{Y} \in \Omega_{(1)}^{4}(M) .
$$

This property determines $\left(\hat{c}_{2}-s^{*} \psi\right)\left(E, \nabla^{E}\right)$ up to exact currents.

The connections $\nabla^{T M}$ and $\nabla^{\bar{E}}$ and the metrics $g$ and $h^{E}$ induce natural Riemannian metrics on the total spaces of $\nu \rightarrow X$ and $\left.\bar{E}\right|_{X} \rightarrow X$. We may assume that $d s: \nu \rightarrow \bar{E}$ and the normal exponential map $\exp _{X}: \nu \rightarrow W$ induce isometries between a neighbourhood of the zero sections in $\nu$ and in $\left.\bar{E}\right|_{X}$ and a neighbourhood of $X$. Then $\left(\left.\bar{E}_{\mathbb{R}}\right|_{X}, \nabla^{E}, h^{E}\right)$ and $\left(\nu,\left.\nabla^{T M}\right|_{\nu},\left.g\right|_{\nu}\right)$ become isomorphic and the splitting $\left.T W\right|_{X}=T X \oplus \nu$ becomes parallel. By (1.6), (1.12), (1.13), and the 
multiplicativity of the $\hat{A}$-form,

$$
\begin{aligned}
\tau_{M}(E)=- & \frac{1}{a_{k+1}} \int_{W} \widehat{A}\left(T W, \nabla^{T W}\right)\left(c_{2} \mathrm{ch}^{\prime}\right)\left(\bar{E}, \nabla^{\bar{E}}\right) \\
& +\frac{1}{a_{k+1}} \int_{M} \widehat{A}\left(T M, \nabla^{T M}\right)\left(\hat{c}_{2} \mathrm{ch}^{\prime}\right)\left(E, \nabla^{E}\right) \\
=- & \frac{1}{a_{k+1}} \int_{X} \widehat{A}\left(T X, \nabla^{T X}\right) \\
& +\frac{1}{a_{k+1}} \int_{M} \widehat{A}\left(T M, \nabla^{T M}\right)\left(\left(\hat{c}_{2}-s^{*} \psi\right) \mathrm{ch}^{\prime}\right)\left(E, \nabla^{E}\right) .
\end{aligned}
$$

Since we have assumed that $M$ and $W$ are spin, there are induced spin structures on $X$ and $Y=X \cap M$. We consider the untwisted Dirac operators $D_{X}$ and $D_{Y}$ with respect to these spin structures. Note that $\Sigma X$ and $\Sigma Y$ carry a quaternionic structure if and only if $\operatorname{dim} X \cong 4 \bmod 8$. But this is the case if and only if $\operatorname{dim} W \cong$ $0 \bmod 8$, that is, if and only if $\Sigma W$ and $\Sigma M$ carry real structures, and hence $D_{M}^{E}$ respects a quaternionic structure on $\Sigma M \otimes E$.

1.10. Proposition. Let $(Y, \nu)$ be a divisor of a quaternionic line bundle $E \rightarrow M$ and assume that a neighbourhood of $Y$ in $M$ is isometric to a neighbourhood of the zero section of $\nu$, where the metric on the total space of $\nu$ is induced by a quaternionic metric and a compatible connection. Let $\alpha_{Y} \in \Omega_{(1)}^{3}(M)$ denote a current with $d \alpha_{Y}=\delta_{Y}$. Then

$$
t_{M}(E)=-\frac{1}{a_{k+1}}\left(\frac{\eta+h}{2}\left(D_{Y}\right)-\int_{M} \widehat{A}\left(T M, \nabla^{T M}\right) \alpha_{Y} \operatorname{ch}^{\prime}\left(E, \nabla^{E}\right)\right) .
$$

If $(M, E)$ bounds, this equation follows from (1.14), (1.15) above and the AtiyahPatodi-Singer index theorem 1.2 applied to the untwisted Dirac operator $D_{X}$ on $X$.

Proof. We use Bismut-Zhang's formula for the $\eta$-invariant of direct images in 8 to derive the result directly from (1.9).

Bismut and Zhang construct a current $\gamma \in \Omega_{(1)}^{\bullet}(M)$ in [8, section 1.c)] such that

$$
d \gamma=2-\operatorname{ch}\left(E, \nabla^{E}\right)-\widehat{A}\left(\nu, \nabla^{\nu}\right)^{-1} \delta_{Y} .
$$

By (1.3), (1.12) and (1.13), the current $\left(s^{*} \psi \mathrm{ch}^{\prime}\right)\left(E, \nabla^{E}\right)-\gamma$ is closed.

Because in our special situation the current $\gamma$ is constructed directly in terms of $s$, there exists a natural current on $E$ whose pullback by $s$ becomes $\gamma$. In particular, we can work with universal currents on $\mathbb{H} P^{N}$ for $N$ sufficiently large. As $\mathbb{H} P^{N}$ has no cohomology in degrees $4 k-1$, we conclude that $\left(s^{*} \psi \operatorname{ch}^{\prime}\right)\left(E, \nabla^{E}\right)-\gamma$ is exact, so that we replace $\gamma$ by $\left(s^{*} \psi \operatorname{ch}^{\prime}\right)\left(E, \nabla^{E}\right)$.

We still assume that $\left.d s\right|_{\nu}$ and the normal exponential map $\exp _{\nu}$ are isometries near the zero section of $\nu \rightarrow Y$. With these assumptions and because $\varepsilon-E$ is a direct image of the trivial complex line bundle on $Y$ as in Remark1.15. Theorem 2.2 in [8] implies that

$$
\begin{aligned}
\frac{\eta+h}{2}\left(D_{M}^{E}\right)-(\eta+h) & \left(D_{M}\right) \\
& =-\frac{\eta+h}{2}\left(D_{Y}\right)-\int_{M} \widehat{A}\left(T M, \nabla^{T M}\right)\left(s^{*} \psi \mathrm{ch}^{\prime}\right)\left(E, \nabla^{E}\right) .
\end{aligned}
$$


Because $H^{3}(M ; \mathbb{Q})=0$, the currents $\alpha_{Y}$ and $\left(\hat{c}_{2}-s^{*} \psi\right)\left(E, \nabla^{E}\right)$ differ by an exact current by (1.14). Together with (1.9), this proves the proposition.

1.d. The relationship with the Adams $e$-invariant. Following the ideas above, we can identify certain cases where the $t$-invariant can be related to the Adams $e$ invariant.

1.11. Proposition. Let $M$ be a stably framed $(4 k-1)$-manifold with $k \geq 3$ and with $H^{3}(M ; \mathbb{Q})=0$. Assume that $E \in \operatorname{Bun}_{0}(M)$ is classified by a map factoring through $\xi: M \rightarrow S^{4} \cong \mathbb{H} P^{1} \subset \mathbb{H} P^{\infty}$ and that $x_{0} \in S^{4}$ is a regular value of $\xi$. If $Y=\xi^{-1}\left(x_{0}\right)$, then $Y$ inherits a stable framing from $M$ and $\xi$, and we have

$$
t_{M}(E)=-e(Y) \text {. }
$$

Proof. By assumption, the manifold $M$ is framed. The framing in particular selects a Chern-Simons type form $\widetilde{A}=\widetilde{A}\left(T M \oplus \mathbb{R}^{N}, \nabla^{0}, \nabla^{T M \oplus \mathbb{R}^{N}}\right)$ such that

$$
\widehat{A}\left(T M, \nabla^{T M}\right)=1+d \widetilde{A} .
$$

We may assume that $Y$ is totally geodesic in $M$. Because the normal bundle to $Y$ is trivialised by $d \xi$, we also have

$$
\widehat{A}\left(T Y, \nabla^{T Y}\right)=1+\left.d \widetilde{A}\right|_{Y} .
$$

Following [5, II, Theorem 4.14], we write the $e$-invariant of $Y$ with the induced framing as

$$
e(Y)=\frac{1}{a_{k+1}}\left(\frac{\eta+h}{2}\left(D_{Y}\right)-\int_{Y} \tilde{A}\right) .
$$

Consider the tautological bundle $\tau \rightarrow \mathbb{H} P^{1}$ and assume that $s_{0}$ is a transversal section with $s_{0}^{-1}(0)=\left\{x_{0}\right\}$. Then $s=\xi^{*} s_{0}$ is a transversal section of $E$ with divisor $Y$. Let us pull $\nabla^{E}$ back from a connection $\nabla^{\tau}$ on $\mathbb{H} P^{1}$; then $\left.\operatorname{ch}^{\prime}\left(E, \nabla^{E}\right)\right|_{Y}=$ 1. From Proposition 1.10 and with $d \alpha_{Y}=\delta_{Y}$, we have

$$
\begin{aligned}
e(Y)+t_{M}(E) & =\frac{1}{a_{k+1}}\left(\int_{M} \widehat{A}\left(T M, \nabla^{T M}\right) \alpha_{Y} \operatorname{ch}^{\prime}\left(E, \nabla^{E}\right)-\int_{Y} \widetilde{A}\right) \\
& =\frac{1}{a_{k+1}} \int_{M}\left(\alpha_{Y} \operatorname{ch}^{\prime}\left(E, \nabla^{E}\right)+d\left(\widetilde{A} \alpha_{Y} \operatorname{ch}^{\prime}\left(E, \nabla^{E}\right)\right)\right)=0,
\end{aligned}
$$

because $\operatorname{ch}^{\prime}\left(E, \nabla^{E}\right)-1 \in \Omega^{4}(M)$ by naturality. Hence $\alpha_{Y} \operatorname{ch}^{\prime}\left(E, \nabla^{E}\right)$ lives in degree $\leq 7<4 k-1$.

The $t$-invariant is clearly unstable from the point of view of $K$-theory, because any invariant that is stable under addition of trivial vector bundles is generated by characteristic classes. On the other hand, the following result shows that the $t$-invariant on $S^{4 k-1}$ is stable in the sense of stable homotopy theory.

1.12. Theorem. Assume that $k \geq 3$. For any homotopy sphere $\Sigma^{4 k-1}$ the homomorphism

$$
t_{\Sigma}: \operatorname{Bun}(\Sigma) \cong \pi_{4 k-2}\left(S^{3}\right) \longrightarrow \mathbb{Q} / \mathbb{Z}
$$

may be identified with the composition

$$
-e \circ S: \pi_{4 k-2}\left(S^{3}\right) \longrightarrow \pi_{4 k-5}^{S} \longrightarrow \mathbb{Q} / \mathbb{Z},
$$

where $-e: \pi_{4 k-5}^{S} \rightarrow \mathbb{Q} / \mathbb{Z}$ and $S: \pi_{4 k-2} \rightarrow \pi_{4 k-5}^{S}$ are respectively the negative of the Adams e-invariant, $e_{\mathbb{R}}^{\prime}$ of [1, Proposition 7.14], and the stabilisation homomorphism. 
1.13. Corollary. The t-invariant defines an injective homomorphism

$$
t_{S^{11}}: \operatorname{Bun}\left(S^{11}\right) \cong \pi_{10}\left(S^{3}\right) \longrightarrow \mathbb{Z} / 15 \text {. }
$$

Proof. In this case stabilisation is injective by [31, Ch. XIII] and the $e$-invariant is injective on the 11-stem by [1, Ex. 7.17].

Proof of Theorem 1.12, Recall that the boundary map associated to the Hopf fibration is a homomorphism $\partial: \pi_{j+1}\left(S^{4}\right) \rightarrow \pi_{j}\left(S^{3}\right)$. Because the fibre $S^{3}$ is contractible in the total space $S^{7}$, the long exact homotopy sequence implies that $\partial$ is surjective.

The Hopf fibration extends to $\mathbb{H} P^{k}$ for all $k$. From the diagram

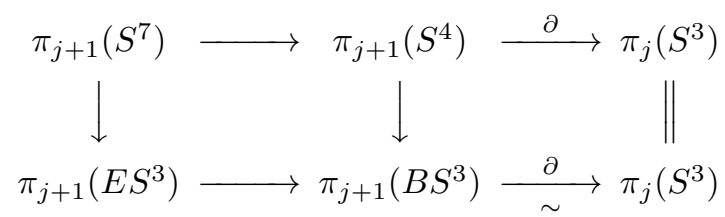

it follows that the homomorphism induced by the standard inclusion

$$
\pi_{j+1}\left(S^{4}\right) \longrightarrow \pi_{j+1}\left(B S^{3}\right) \cong \pi_{j}\left(S^{3}\right)
$$

is onto. So without loss of generality, the quaternionic line bundle $E \rightarrow S^{4 k-1}$ is classified by a map $\xi: S^{4 k-1} \rightarrow S^{4} \subset B S^{3}$. Note that $E \cong \xi^{*} H$, where $H$ is the tautological bundle over $S^{4}$.

We give $S^{4 k-1}$ the standard stable framing and consider the class

$$
[\xi] \in \pi_{4 k-5}^{S} \cong \Omega_{4 k-5}^{\mathrm{fr}} \cong \Omega_{4 k-1}^{\mathrm{fr}}\left(S^{4}\right),
$$

where $\Omega_{*}^{\text {fr }}$ denotes the framed bordism and we have applied the Pontryagin-Thom isomorphism. By the Pontryagin-Thom construction, this class $[\xi]$ is represented by the quaternionic divisor $Y=\xi^{-1}\left(x_{0}\right)$ for some regular value $x_{0} \in S^{4}$ of $\xi$. In particular, the manifold $Y$ inherits a framing from $\xi$. Then by Proposition 1.11, we have

$$
t_{S^{4 k-1}}(E)=-e(Y)=-e([\xi]) .
$$

1.14. Remark. Theorem 1.12 is void for $k=1$ and it does not hold for $k=2$. To consider the case $k=2$ we first record some well-known facts about the Hopf fibration:

$$
p_{2}: S^{7} \rightarrow S^{4}, \quad(x, y) \mapsto[x, y] .
$$

Here $(x, y) \in S^{7} \subset \mathbb{R}^{4} \times \mathbb{R}^{4}$, and $[x, y]$ describes a point in $\mathbb{H} P^{1}=S^{4}$ using homogeneous coordinates. From this description it is a simple matter to verify that $p_{2}$ is the quotient map of a principal $S^{3}$-bundle with characteristic map

$$
\sigma: S^{3} \rightarrow S O(4), x \mapsto(v \mapsto x \cdot v),
$$

where $v \in \mathbb{H}$ and $x \cdot v$ denotes quaternionic multiplication. In the notation of 30 , $22.6 \& 22.7$ ], $\sigma$ defines the homotopy class $\beta_{3} \in \pi_{3}(S O(4))$ and by [30, 23.7] the stabilisation of $\beta_{3}$, denoted by $\beta$, generates $\pi_{3}(S O) \cong \mathbb{Z}$.

Next recall the $J$-homomorphism, $J_{r, n}: \pi_{r}\left(O_{n}\right) \rightarrow \pi_{n+r}\left(S^{n}\right)$ as defined in 35 , Example 2, p. 504]. We leave it for the reader to verify that the map $p_{2}$ above may be identified with the map $J_{3,4}(\sigma)$. Hence, if $\left(S^{3}, \pi_{H}\right)$ is a fibre of the Hopf fibration $p_{2}$ with the induced framing, then the framed bordism class $\left[S^{3}, \pi_{H}\right]$ is given by

$$
\left[S^{3}, \pi_{H}\right]=J(\beta) \in \pi_{3}^{s},
$$


where $\beta$ is the generator $\pi_{3}(S O)$ defined above and $J: \pi_{3}(S O) \rightarrow \pi_{3}^{s}$ is the stable $J$-homomorphism.

Now consider the composite map

$$
S^{7} \stackrel{p_{2}}{\longrightarrow} S^{4} \stackrel{F_{c}}{\longrightarrow} S^{4},
$$

where $F_{c}$ is a self-map of degree $c \in \mathbb{Z}$. Let $E_{c} \rightarrow S^{7}$ be the pullback of the tautological bundle $H \rightarrow \mathbb{H} P^{1}$ along $F_{c} \circ p_{2}$. By Example 3.5.

$$
t_{S^{7}}\left(E_{c}\right)=\frac{c(c-1)}{24} \in \mathbb{Q} / \mathbb{Z} .
$$

On the other hand, let $x_{0} \in S^{4}$ be a regular value of $F_{c}$ and let $Y_{c} \subset S^{7}$ be its preimage: $Y_{c}$ is a framed 3-manifold which is a disjoint union of $c$ copies $\left(S^{3}, \pi_{H}\right)$, the framed fibre of the Hopf fibration. Now by Proposition 1.11 the proof of Theorem 1.12 and (1.17) we have

$$
S\left(\left[\xi_{c}\right]\right)=\left[Y_{c}\right]=c \cdot\left[S^{3}, \pi_{H}\right]=c \cdot J(\beta) \in \pi_{3}^{s},
$$

where $\xi_{c} \in \pi_{6}\left(S^{3}\right)$ is the homotopy class of the clutching function for $E_{c}$.

Now by [1, Ex. 7.17], $e(J(\beta))= \pm 1 / 24$ and so

$$
e \circ S\left(\xi_{c}\right)=\frac{ \pm c}{24}
$$

Comparing (1.18) and (1.19) we deduce that $t_{S^{7}}\left(E_{c}\right) \neq-e \circ S\left(\left[\xi_{c}\right]\right)$ in general.

1.15. Remark. From a more technical point of view, Theorem 1.12 fails for $k=2$ because there are different possible correction terms that construct a differential topological invariant out of the $\eta$-invariant of the untwisted Dirac operator on the divisor $Y$, leading to different invariants. This is particularly interesting from Bunke's more general point of view; see [10], section 5.5.

There is a fundamental difference between the class $\hat{c}_{2} \in \Omega^{3}(M) / \mathrm{im} d$ and the currents $s^{*} \psi$ and $\gamma \in \Omega_{(1)}^{3}(M)$. The latter are natural in $E$ and $s$ as we have seen in the proof above. In particular, we can choose $s$ in such a way that $\|s\|=1$ outside a small neighbourhood $U$ of $Y$. This allows us to choose $\nabla^{E}$ in such a way that $\nabla^{E} s=0$ outside $U$. In other words, contributions coming from $s^{*} \psi$ or $\gamma$ localise near $Y$ and do not involve any other topological information about $M$.

On the other hand, the class $\hat{c}_{2}$ is constructed using the assumption that $c_{2}(E)$ is torsion and that $H^{3}(M ; \mathbb{Q})=0$. In particular, the class $\hat{c}_{2}$ is not natural in $E$, but depends on the topology of $M$.

\section{The $t$-INVARIANT OF 7 -MANIFOLDS}

In this section we investigate the $t$-invariant in dimension 7: in Section 2.a we show that the $t$-invariant classifies closed smooth 2-connected rational homology 7 spheres up to connected sum with homotopy spheres. In Section 2.b we show that the $t$-invariant detects homeomorphisms which are not homotopic to PL homeomorphisms on 2-connected rational homology 7 -spheres. In Section 2.c we relate the $t$-invariant to the $s$-invariants of Kreck and Stolz 25] and their generalisations in Hepworth [23]. 
2.a. Classification results for 2-connected 7-manifolds. Throughout this subsection $M$ shall be a closed smooth oriented 2-connected 7 -manifold. In addition we assume that $M$ is a rational homology sphere which is equivalent to assuming that $\pi_{3}(M) \cong H_{3}(M) \cong H^{4}(M)$ are finite groups.

We first recall the classification of such 2-connected rational homology spheres started in [36 and completed in [12. We then relate the $t$-invariant from Section 1 to this classification to obtain a classification theorem for such manifolds $M$ using $t_{M}$ and $\mu(M)$, the Eells-Kuiper invariant of $M$, which we recall below.

Recall that $\Theta_{7}$ denotes the group of diffeomorphism classes of oriented homotopy 7 -spheres and that $\Theta_{7} \cong \mathbb{Z} / 28$. The homotopy 7 -spheres can be detected by the Eells-Kuiper invariant $\mu(\Sigma) \in \mathbb{Q} / \mathbb{Z}$ which by [17, $\S 6]$ defines an injective homomorphism

$$
\mu: \Theta_{7} \longrightarrow \mathbb{Q} / \mathbb{Z}
$$

Moreover the definition of $\mu$ can be extended to any 2-connected rational homology 7-sphere $M$ to give $\mu(M) \in \mathbb{Q} / \mathbb{Z}$ with

$$
\mu(M \sharp \Sigma)=\mu(M)+\mu(\Sigma) \in \mathbb{Q} / \mathbb{Z} .
$$

The definition of $\mu(M)$ in this case is by now routine: from the analytic point of view it can be found in [16, 25]; using coboundaries, the details are in [12, 2.12], where $\mu$ is called $s_{1}$. With either definition the additivity in (2.1) is clear.

This gives an effective strategy for classifying 2-connected rational homology 7-spheres. First, consider these manifolds up to almost diffeomorphism, where an almost diffeomorphism is a homotopy sphere $\Sigma$ and a diffeomorphism $f: N \sharp \Sigma \cong M$. If $M$ and $N$ are almost diffeomorphic, then they are diffeomorphic if and only if $\mu(M)-\mu(N)=\mu(\Sigma)=0$.

We turn to consider almost diffeomorphism invariants of $M$. Since $M$ is 2connected it possesses a unique equivalence class of spin structures. An important invariant is the first characteristic class of spin manifolds which generates the group $H^{4}(B$ Spin $) \cong \mathbb{Z}$. We choose the generator $p \in H^{4}(B$ Spin $)$ so that $2 p=p_{1}$, where $p_{1}$ is the first Pontryagin class. The class $p$ is often called "half the first Pontryagin class" and is sometimes denoted $\frac{p_{1}}{2}$. For any spin manifold $X$, we have $p_{X}=p(T X) \in H^{4}(X)$.

An important fact about closed smooth 2-connected 7-manifolds proven in 36, Theorem 4] is that every one is the boundary of a handlebody $W$ : i.e. $W$ is a 3 connected 8-manifold obtained from $D^{8}$ by attaching 4 -handles, and the boundary of $W$ is identified with $M$. We consider the exact sequence

$$
0 \longrightarrow H^{4}(W, M) \longrightarrow H^{4}(W) \stackrel{i^{*}}{\longrightarrow} H^{4}(M) \longrightarrow 0,
$$

which allows one to define the following quadratic linking function

$$
q_{M}: H^{4}(M) \longrightarrow \mathbb{Q} / \mathbb{Z}, \quad x \longmapsto \frac{1}{2}\left(\left(\bar{x}+p_{W}\right) \smile \hat{x}\right)[W, M],
$$

where $\bar{x} \in H^{4}(W)$ maps to $x \in H^{4}(M)$ and $\hat{x} \in H^{4}(W, M ; \mathbb{Q})$ maps to $\bar{x}$ under the isomorphism $H^{4}(W, M ; \mathbb{Q}) \cong H^{4}(W ; \mathbb{Q})$.

2.1. Lemma (cf. [12, Lemma 2.51]). The function $q_{M}$ is a well-defined almost diffeomorphism invariant of $M$.

We say that $q_{M}$ and $q_{N}$ are isomorphic if there is an isomorphism $A: H^{4}(M) \cong$ $H^{4}(N)$ such that $q_{M}=q_{N} \circ A$. The following is also proven in [12. 
2.2. Theorem (cf. [12, Theorem A]). Let $N$ and $M$ be 2-connected rational homology 7-spheres.

(1) There is an almost diffeomorphism $f: N \sharp \Sigma \cong M$ with induced map $f^{*}=$ $A: H^{4}(M) \cong H^{4}(N)$ if and only if $q_{M}=q_{N} \circ A$.

(2) $M$ is diffeomorphic to $N$ if and only if $q_{M}$ is isomorphic to $q_{N}$ and $\mu(M)=$ $\mu(N)$.

2.3. Remark. The linking form of $M$

$$
b_{M}: H^{4}(M) \times H^{4}(M) \longrightarrow \mathbb{Q} / \mathbb{Z}
$$

is a non-singular symmetric pairing (see [32, $\S 12 \mathrm{~A}]$ for a definition). In the notation of (2.3), we have

$$
b_{M}(x, y)=(\bar{x} \smile \hat{y})[W, M]=(\hat{x} \smile \bar{y})[W, M] .
$$

From (2.3) we see that $q_{M}$ refines $b_{M}$ in the following sense:

$$
q_{M}(x+y)=q_{M}(x)+q_{M}(y)+b_{M}(x, y) \quad \forall x, y \in H^{4}(M) .
$$

We also see that $q_{M}$ need not be homogeneous; i.e. $q_{M}(x) \neq q_{M}(-x)$ in general. However, the homogeneity defect of $q$ is determined by $p_{M}$ :

$$
q_{M}(x)-q_{M}(-x)=\left(p_{W} \smile \hat{x}\right)[W, M]=b_{M}\left(p_{M}, x\right) .
$$

Now recall that $\operatorname{Bun}(M)$ denotes the set of isomorphism classes of principal $S^{3}$-bundles over $M$. We have an action of $\operatorname{Bun}\left(S^{7}\right) \cong \pi_{6}\left(S^{3}\right) \cong \mathbb{Z}_{12}$ on $\operatorname{Bun}(M)$ by

$$
\operatorname{Bun}(M) \times \operatorname{Bun}\left(S^{7}\right) \longrightarrow \operatorname{Bun}(M), \quad(E, F) \longmapsto E \sharp F,
$$

which is defined by cutting and regluing a given principal $S^{3}$-bundle $E$ over $M$ along $S^{6}=\partial D^{7} \subset M$ using the clutching function of $F$. Next we relate $q_{M}$ to the $t$-invariant $t_{M}$ of Definition 1.4

2.4. Theorem. Let $M$ be a 2-connected rational homology 7-sphere.

(1) For $M=S^{7}$, the $t$-invariant defines an injective homomorphism

$$
t_{S^{7}}: \operatorname{Bun}\left(S^{7}\right) \cong \mathbb{Z} / 12 \subset \mathbb{Q} / \mathbb{Z} .
$$

(2) The group $\operatorname{Bun}\left(S^{7}\right)$ acts freely on $\operatorname{Bun}(M)$ with

$$
\operatorname{Bun}(M) / \operatorname{Bun}\left(S^{7}\right) \underset{c_{2}}{\stackrel{c_{2}}{\longrightarrow}} H^{4}(M),
$$

and for all $(E, F) \in \operatorname{Bun}(M) \times \operatorname{Bun}\left(S^{7}\right)$ we have

$$
t_{M}(E \sharp F)=t_{M}(E)+t_{S^{7}}(F) .
$$

(3) For all $E \in \operatorname{Bun}(M)$ there is an equality

$$
q_{M}\left(c_{2}(E)\right)=12 t_{M}(E) \in \mathbb{Q} / \mathbb{Z} ;
$$

using (2), it follows that the t-invariant determines $q_{M}$.

As an immediate consequence of Theorem 2.2 (2) and Theorem 2.4 (3) we have

2.5. Corollary. Let $N$ and $M$ be 2-connected rational homology 7-spheres. Then $N$ is diffeomorphic to $M$ if and only if $t_{N}$ is isomorphic to $t_{M}$ and $\mu(N)=\mu(M)$. 
Proof of Theorem 2.4. For (1) we use the bundle $E:=E_{1,1,1} \rightarrow M_{1,1}$ from Proposition 2.6 below. The manifold $M_{1,1} \cong S^{7}$ is the total space of the Hopf fibration and so $E$ is a bundle over $S^{7}$ with $t_{S^{7}}(E)=\frac{1}{12}$. By Proposition 1.5 (2), $t_{S^{7}}$ is a homomorphism with $t_{S^{7}}\left(E^{\sharp k}\right)=\frac{k}{12}$.

For part (2), let $W$ be a handlebody with $\partial W=M$ as above. As $W$ is homotopy equivalent to a wedge of 4-spheres and $B S^{3}$ is three-connected, we have an isomorphism $c_{2}: \operatorname{Bun}(W) \rightarrow H^{4}(W)$. Hence $c_{2}: \operatorname{Bun}(M) \rightarrow H^{4}(M)$ is onto by (2.2). There is a homotopy equivalence $M \simeq M^{\bullet} \cup e^{7}$, where $M^{\bullet}:=M-\operatorname{int}\left(D^{7}\right)$ is homotopy equivalent to a degree 3 Moore space: i.e. the reduced homology of $M^{\bullet}$ vanishes except in degree 3 and $M^{\bullet}$ is homotopy equivalent to a $C W$-complex with cells only in dimensions 3 and 4 . Because $S^{4}=\mathbb{H} P^{1} \subset B S^{3}$ is a 5-skeleton for $B S^{3}$ we see that $\left[M^{\bullet}, B S^{3}\right]=\left[M^{\bullet}, S^{4}\right]$ and since $M^{\bullet}$ is a degree 3 Moore space the primary obstruction to null-homotopy defines a bijection $\left[M^{\bullet}, S^{4}\right] \cong H^{4}\left(M^{\bullet}\right)$. Moreover, since the restriction of $c_{2}$ to $\mathbb{H} P^{1}$ generates $H^{4}\left(\mathbb{H} P^{1}\right)$ we see that the map $c_{2}: \operatorname{Bun}\left(M^{\bullet}\right) \rightarrow H^{4}\left(M^{\bullet}\right) \cong H^{4}(M)$ is a bijection. By the surjectivity of $c_{2}: \operatorname{Bun}(M) \rightarrow H^{4}(M)$, each quaternionic line bundle $E^{\bullet}$ on $M^{\bullet}$ extends to $M$, so $\left.E^{\bullet}\right|_{\partial D^{7}}$ is trivial and $\operatorname{Bun}\left(S^{7}\right)$ acts transitively on the set of possible extensions.

The formula for $t_{M}$ given in (2) is a special case of the additivity formula of Proposition [1.5 (2). Together with (11), it proves that the action of $\operatorname{Bun}\left(S^{7}\right)$ on $\operatorname{Bun}(M)$ is free.

For part (3), let $\bar{E} \rightarrow W$ be a quaternionic line bundle with $c_{2}(\bar{E})=\bar{x} \in H^{4}(W)$. Let $\hat{c}_{2}(\bar{E}) \in H^{4}(W, M ; \mathbb{Q})$ be a lift of $c_{2}(\bar{E})$. We use equation (1.6), the fact that the $\widehat{A}$-genus begins as $\widehat{A}=1-\frac{p}{12}+\ldots$ and (1.4) to deduce the following formula:

$$
t_{M}\left(i^{*} E\right)=\frac{1}{24}\left(\hat{c}_{2}(\bar{E}) \smile\left(p_{W}+c_{2}(\bar{E})\right)\right)[W, M] \in \mathbb{Q} / \mathbb{Z} .
$$

Comparing (2.5) with (2.3) we see that $q_{M}\left(c_{2}(E)\right)=12 t_{M}(E)$.

We finish this subsection by recording calculations of $t_{M}$ in some examples. Let $n$ and $p$ be integers with $n \neq 0$ and let $\pi: W_{n, p} \rightarrow S^{4}$ be the disc bundle of a vector bundle over the 4 -sphere with Euler class $e(\pi)=n \bar{x}$ and with $p_{W}=p \bar{x}$ : here we fix a generator $\bar{x}$ of $H^{4}\left(S^{4}\right)$ and identify $H^{4}\left(S^{4}\right)=H^{4}\left(W_{n, p}\right)$. Also let $i: M_{n, p} \rightarrow W_{n, p}$ be the inclusion of the boundary so that $M_{n, p}$ is the total space of a 3 -sphere bundle over $S^{4}$. We remark that these total spaces were classified up to diffeomorphism, homeomorphism and homotopy equivalence in [13.

2.6. Proposition. With $i: M_{n, p} \rightarrow W_{n, p}$ and $\bar{x} \in H^{4}\left(S^{4}\right)$ as above and $k$ any integer, let $\bar{E}_{n, p, k} \rightarrow W_{n, p}$ be the quaternionic line bundle with $c_{2}(\bar{E})=k \bar{x}$ and let $E_{n, p, k}:=i^{*} \bar{E}_{n, p, k}$. Then for $M=M_{n, p}$ we have

$$
t_{M}\left(i^{*} E_{n, p, k}\right)=\frac{k(p+k)}{24 n} \in \mathbb{Q} / \mathbb{Z} .
$$

Proof. The intersection form $\left(H_{4}\left(W_{n, p}\right), \lambda\right)$ is isomorphic to $(\mathbb{Z}, n)$; see [13, $\S 3$, p. 368], noting that $M_{n, p}$ above is diffeomorphic to the total space $M_{m, n}$ of [13], where $p=n+2 m$. We now simply apply the expression for $t_{M}$ in (2.5).

2.7. Remark. While it is possible to use the analytic definition of the $t$-invariant to compute $t_{M}$ in this case, the computations still require sophisticated techniques and are somewhat lengthy compared to the topological definition. 
Searching for new compact Riemannian manifolds of positive sectional curvature in [22], Grove, Wilking and Ziller considered two families $M_{\left(p_{-}, q_{-}\right),\left(p_{+}, q_{+}\right)}$ and $N_{\left(p_{-}, q_{-}\right),\left(p_{+}, q_{+}\right)}$of seven-manifolds of cohomogeneity one. The first family consists of two-connected manifolds, while the second is of the type considered by Kreck and Stolz in 25]; see also Remark 2.18 below. By [22], the manifolds $P_{n}=M_{(1,1),(2 n-1,2 n+1)}$ are the only members of the $M$-family that can carry metrics of positive sectional curvature. Note that $P_{1}=S^{7}$, and that $P_{2}$ is homeomorphic to the unit tangent bundle of $S^{4}$, which is diffeomorphic to the principal bundle $M_{2,2}$ of Proposition 2.6. It was then proved independently by Dearricott [14] and Grove, Verdiani and Ziller [21] that $P_{2}$ carries a metric of positive sectional curvature. In [19], the second author computed the $t$-invariants of sufficiently many quaternionic line bundles over $M_{\left(p_{-}, q_{-}\right),\left(p_{+}, q_{+}\right)}$to determine its almost-diffeomorphism type using Corollary 2.5 above.

2.8. Example. For each space $P_{n}$, there exists a family of quaternionic line bundles $E_{k} \rightarrow P_{n}$ with $c_{2}\left(E_{k}\right) \equiv k \in H^{4}\left(P_{n}\right) \cong \mathbb{Z} / n$ and

$$
t_{P_{n}}\left(E_{k}\right)=\frac{k(k+n)}{24 n}+\frac{k(k-1)(n-1)}{6} \in \mathbb{Q} / \mathbb{Z}
$$

by [19, section 3.e]. Hence, we have $q_{P_{n}}(k)=\frac{k(k+n)}{2 n}$.

By Theorems 2.2 and 2.4 and Proposition 2.6 above, the spaces $P_{n}$ are almost diffeomorphic to the manifolds $M_{n, n}$ of Proposition 2.6, in other words, to the total spaces of the principal $S^{3}$-bundles over $S^{4}$ with Euler class given by $n$. The classification is then completed by computing the Eells-Kuiper invariant $\mu\left(P_{n}\right)$. Let $\Sigma$ be a generator of $\Theta_{7}$ with $\mu(\Sigma)=\frac{1}{28}$. Then [19, Theorem 0.3] states that

$$
P_{n} \cong M_{n, n} \sharp \Sigma^{\sharp \frac{n-n^{3}}{6}} \text {. }
$$

2.b. Detecting exotic homeomorphisms. Throughout this subsection we assume that $M$ and $N$ are closed smooth oriented 2-connected rational homology 7-spheres and that all maps preserve orientation. We call a homeomorphism

$$
h: N \cong M
$$

exotic if it is not homotopic to a piecewise linear (PL) homeomorphism. The main result of this section is that $h$ is exotic if and only if the induced map $h^{*}: \operatorname{Bun}(M) \rightarrow$ $\operatorname{Bun}(N)$ does not preserve the $t$-invariants of $M$ and $N$.

As we explain below (see (2.6), Lemma 2.10 and Definition 2.13), the following is a consequence of the theory of topological surgery: For any homeomorphism $h: N \cong$ $M$ there is an invariant

$$
\kappa(h) \in H^{3}(M ; \mathbb{Z} / 2),
$$

which depends only on the homotopy class of $h$, such that $h$ is exotic if and only if $\kappa(h) \neq 0$.

2.9. Theorem. A homeomorphism $h: N \rightarrow M$ is exotic if and only if

$$
t_{M} \neq t_{N} \circ h^{*} .
$$

More precisely, for all $E \in \operatorname{Bun}(M)$,

$$
\left(\kappa(h) \smile c_{2}(E)\right)[M]_{2}=t_{N}\left(h^{*} E\right)-t_{M}(E) \in \mathbb{Z} / 2 \subset \mathbb{Q} / \mathbb{Z},
$$

where $[M]_{2}$ generates $H_{7}(M ; \mathbb{Z} / 2)$.

We begin by showing that $t_{M}$ is invariant under PL-homeomorphisms. 
2.10. Lemma. If $h: N \cong M$ is a PL-homeomorphism, then $t_{M}=t_{N} \circ h^{*}$.

Proof. It follows from smoothing theory, see [12, $\S 6$, Theorem 6.1], that there is a homotopy 7 -sphere $\Sigma$ such that $h$ is homotopic to a diffeomorphism $g: N \sharp \Sigma \rightarrow M$. The lemma now follows by Proposition 1.5 (3).

2.11. Remark. Note that [12, Theorem 6.1] also proves that $N$ is homeomorphic to $M$ if and only if $N$ is $P L$-homeomorphic to $M$. Hence the function $t_{M}$ is a topological invariant in the weak sense that if $h: N \rightarrow M$ is a homeomorphism, then there is a homeomorphism, indeed a $P L$-homeomorphism, $g: N \rightarrow M$ such that $t_{M}=t_{N} \circ g^{*}$.

Proof of Theorem 2.9. We will not explicitly construct exotic homeomorphisms but rather we use surgery theory to show that they exist. Hence we begin by briefly recalling some essential notions from surgery. Let $C a t=T o p$ or $P L$ denote respectively the topological and piecewise linear categories of manifolds. Recall the Cat-structure set of $M, \mathcal{S}^{C a t}(M)$, which consists of equivalence classes of structures which are homotopy equivalences $g: N \rightarrow M$ :

$$
\mathcal{S}^{C a t}(M):=\{g: N \simeq M \mid N \text { a Cat-manifold }\} / \sim .
$$

Two structures $g_{0}$ and $g_{1}$ are equivalent if $g_{1} \circ g_{0}^{-1}$ is homotopic to a Cat-isomorphism. The base-point of $\mathcal{S}^{C a t}(M)$ is the trivial element [Id: $M \rightarrow M$ ].

The Cat-structure set of $M$ lies in the Cat-surgery exact sequence (see 33 , Chapter 10], [26, p.330] for more definitions and details):

$$
L_{8}(e) \longrightarrow \mathcal{S}^{C a t}(M) \stackrel{\eta^{C a t}}{\longrightarrow}[M, G / C a t] \longrightarrow L_{7}(e) .
$$

Here $L_{7}(e)=0$ and $L_{8}(e) \cong \mathbb{Z}$ are the simply connected surgery obstruction groups, $G=\lim _{n} \operatorname{Map}_{ \pm 1}\left(S^{n}, S^{n}\right)$ is the monoid of stable self-equivalences of the $n$-sphere, Top and $P L$ are the groups of stable homeomorphisms, respectively PLhomeomorphisms, of Euclidean space and $\eta^{\text {Cat }}$ denotes the Cat-normal invariant map. It is well known that the map $L_{8}(e) \rightarrow \mathcal{S}^{C a t}(M)$ vanishes in both the topological or piecewise linear categories; see [9, II.4.10 \& II.4.11] for the PL case, which implies the topological case. So from the Cat-surgery exact sequences we obtain the following commuting square:

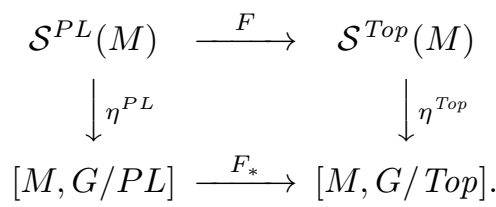

Here each $\eta^{C a t}$ is a bijection, $F$ is the forgetful map and the canonical map $G / P L \rightarrow$ $G /$ Top induces $F_{*}$.

2.12. Lemma. Let $M$ be a 2-connected rational homology 7-sphere. Then there are bijections $\mathcal{S}^{P L}(M) \equiv H^{4}(M)$ and $\mathcal{S}^{T o p}(M) \equiv H^{4}(M)$ such that the forgetful map $F$ corresponds to the map $\times 2$ in the cohomology Bockstein sequence for the coefficient sequence $0 \rightarrow \mathbb{Z} \rightarrow \mathbb{Z} \rightarrow \mathbb{Z} / 2 \rightarrow 0$. In particular, there is a short exact sequence of abelian groups

$$
0 \longrightarrow H^{3}(M ; \mathbb{Z} / 2) \longrightarrow \mathcal{S}^{P L}(M) \stackrel{F}{\longrightarrow} \mathcal{S}^{T o p}(M) \longrightarrow H^{4}(M ; \mathbb{Z} / 2) \longrightarrow 0 .
$$


Proof. Using (2.6) it suffices to calculate $F_{*}:[M, G / P L] \rightarrow[M, G / T o p]$, which is routine: since $M$ is a 2 -connected 7 -manifold it has a $C W$-decomposition with cells in dimensions $0,3,4$ and 7 and so by [34, Corollary 2.3.1] there is a homotopy equivalence

$$
M \simeq M^{\bullet} \cup_{\phi} e^{7},
$$

where $M^{\bullet}:=M-\operatorname{int}\left(D^{7}\right), \phi$ is the attaching map for the top cell of $M$, and $M^{\bullet}$ is homotopy equivalent to the Moore space $M(H, 3)$, where $H=H_{3}(M)$. We write $H=\bigoplus_{i=1}^{r} C_{k(i)}$ as a sum of cyclic groups and so we have a homotopy equivalence

$$
M^{\bullet} \simeq \bigvee_{i=1}^{r} M(\mathbb{Z} / k(i), 3) \simeq \bigvee_{i=1}^{r}\left(S_{i}^{3} \cup_{k(i)} e_{i}^{4}\right),
$$

where $k(i)$ denotes a map $S_{i}^{3} \rightarrow S_{i}^{3}$ of degree $k(i)$.

It is well known (see e.g. [27, Corollay $2.24 \&$ Remark 2.25]) that the lowdimensional homotopy groups of $G / P L$ are given by

$$
\pi_{i}(G / P L) \cong 0,0, \mathbb{Z} / 2,0, \mathbb{Z}, \quad i=0,1,2,3,4,
$$

and that the induced homomorphism $F_{*}: \pi_{i}(G / P L) \rightarrow \pi_{i}(G / T o p)$ is an isomorphism in all dimensions except for $i=4$ when it is isomorphic to the homomorphism $\times 2: \mathbb{Z} \rightarrow \mathbb{Z}$. Since $M^{\bullet}$ has cells only in dimensions 0,3 and 4 , a simple application of obstruction theory shows that the homomorphism $\left[M^{\bullet}, G / P L\right] \rightarrow$ $\left[M^{\bullet}, G / T o p\right]$ is isomorphic to the homomorphism $\times 2: H^{4}\left(M^{\bullet}\right) \rightarrow H^{4}\left(M^{\bullet}\right)$.

It remains to show that the inclusion $M^{\bullet} \rightarrow M$ of the 4-skeleton induces isomorphisms $[M, G / C a t] \cong\left[M^{\bullet}, G / C a t\right]$. Let

$$
C: M^{\bullet} \longrightarrow \bigvee_{i=1}^{r} S^{4}
$$

be the map collapsing the 3-skeleton and observe that the homomorphism

$$
C^{*}: \bigoplus_{i=1}^{r} \pi_{4}(G / C a t) \longrightarrow\left[M^{\bullet}, G / C a t\right]
$$

is onto because $\pi_{3}(G / C a t)=0$. It follows that the induced homomorphism

$$
\phi^{*}:\left[M^{\bullet}, G / C a t\right] \longrightarrow \pi_{6}(G / C a t)
$$

vanishes since the composite $C \circ \phi: S^{6} \rightarrow \vee S^{4}$ must be a wedge of trivial maps or the essential map $\eta^{2}: S^{6} \rightarrow S^{4}$ and the induced homomorphism $\left(\eta^{2}\right)^{*}: \pi_{4}($ G/Cat $) \rightarrow$ $\pi_{6}(G / C a t)$ factors through $\pi_{5}(G / C a t)=0$. From the short exact sequence

$$
0=\pi_{7}(G / C a t) \longrightarrow[M, G / C a t] \longrightarrow\left[M^{\bullet}, G / C a t\right] \stackrel{\phi^{*}}{\longrightarrow} \pi_{6}(G / C a t)
$$

we deduce that $[M, G / C a t] \rightarrow\left[M^{\bullet}, G / C a t\right]$ is an isomorphism.

Observing that a homeomorphism $h: N \cong M$ defines the element $[h] \in \mathcal{S}^{P L}(M)$ such that $F([h])=[\mathrm{Id}] \in \mathcal{S}^{T o p}(M)$ we make the following

2.13. Definition. Let $h: N \rightarrow M$ be a homeomorphism. Define

$$
\kappa(h):=[h] \in H^{3}(M ; \mathbb{Z} / 2),
$$

where we use Lemma 2.12 and the bijections $\eta^{C a t}$ of (2.6) to identify

$$
H^{3}(M ; \mathbb{Z} / 2)=F^{-1}([\mathrm{Id}]) \subset \mathcal{S}^{P L}(M) .
$$


By definition we have $[h] \neq 0$ if and only if $h$ is exotic. Moreover $H^{3}(M ; \mathbb{Z} / 2)=$ $H^{4}(M ; \mathbb{Z} / 2)=0$ if and only if $H^{4}(M)$ contains no 2-torsion. In this case the forgetful map $F: \mathcal{S}^{P L}(M) \rightarrow \mathcal{S}^{T o p}(M)$ is a bijection and $M$ admits no exotic homeomorphisms. Henceforth we assume that $H^{4}(M)$ contains 2-torsion.

2.14. Lemma. Let $x \in H^{3}(M ; \mathbb{Z} / 2)$. Then the manifold $M$ admits a self-homotopy equivalence $p(x): M \simeq M$ such that:

(1) the $P L$ normal invariant of $p(x)$ is $x \in[M, G / P L]$,

(2) $t_{M}\left(p(x)^{*} E\right)-t_{M}(E)=\left(x \smile c_{2}(E)\right)[M]_{2}$ for all $E \in \operatorname{Bun}(M)$.

Before proving Lemma 2.14let us complete the proof of Theorem2.9. Let $h: N \simeq$ $M$ be a homeomorphism. If $h$ is not exotic, then $t_{M}=t_{N} \circ h^{*}$ by Lemma 2.10. Conversely, suppose that $h$ is exotic: the $P L$ normal invariant of $h$ is a non-zero element $x \in H^{3}(M ; \mathbb{Z} / 2)$. By Lemma 2.14(1), we have $[h]=[p(x)] \in \mathcal{S}^{P L}(M)$ and so by definition there is a $P L$ homeomorphism $d: N \rightarrow M$ such that $h \simeq p(x) \circ d$. It follows for all $E \in \operatorname{Bun}(M)$ that

$$
t_{N}\left(h^{*} E\right)=t_{N}\left(d^{*} p(x)^{*} E\right)=t_{M}\left(p(x)^{*} E\right)=t_{M}(E)+\left(x \smile c_{2}(E)\right)[M]_{2} .
$$

Here we used Lemma 2.10 for the second equality and Lemma 2.14(2) for the third equality. Finally, note that $p(x)$ has a trivial topological normal invariant and so is homotopic to an exotic self-homeomorphism $h: M \cong M$. This completes the proof of Theorem 2.9.

Proof of Lemma 2.14. We begin with the definition of $p(x)$ and the proof of part (1). Recall that $M^{\bullet}:=M-\operatorname{int}\left(D^{7}\right)$ and that $M^{\bullet}$ is homotopy equivalent to the Moore space $M(H, 3)$, where $H=H_{3}(M) \cong \bigoplus_{i=1}^{r} C_{k(r)}$ is a sum of cyclic groups. We assume that $k(r)$ is even if and only if $i \in\{1, \ldots, s\}$.

The self-equivalences $p(x): M \rightarrow M$ we construct will be pinch maps. Pinch maps are described in [28, §4] for manifolds with boundary but to begin we shall consider the closed case. Take an element $\psi \in \pi_{7}\left(M^{\bullet}\right)$ and define $p(\psi)$ to be the composition

$$
p(\psi):=(\operatorname{Id} \vee \psi) \circ p: M \longrightarrow M \vee S^{7} \longrightarrow M,
$$

where $p: M \rightarrow M \vee S^{7}$ collapses the boundary of a 7-disc $D^{7} \subset M$ to a point and we identify $M=M / D^{7}$.

To build pinch maps $p(\psi)$ we shall need some knowledge of $\pi_{7}\left(M^{\bullet}\right)$ : recall that $C: M^{\bullet} \rightarrow \bigvee_{i=1}^{r} S_{i}^{4}$ collapses the 3 -skeleton and that $\pi_{7}\left(S_{i}^{4}\right) \cong \mathbb{Z} \oplus \mathbb{Z} / 12$. Observe also that $M^{\bullet}=S(M(H, 2))$ is the suspension of the degree two Moore space.

2.15. Lemma. For $i=1, \ldots, s$ there are homotopy classes $\varphi_{i} \in \pi_{6}(M(H, 2))$ such that the suspensions $\psi_{i}:=S\left(\varphi_{i}\right) \in \pi_{7}\left(M^{\bullet}\right)$ satisfy

$$
C_{*}\left(\psi_{i}\right)=(0,6) \in \mathbb{Z} \times \mathbb{Z} / 12 \cong \pi_{7}\left(S_{i}^{4}\right) \subset \pi_{7}\left(\bigvee_{i=1}^{r} S_{i}^{4}\right)
$$


Proof. The stabilisation homomorphism $S: \pi_{6}\left(M(H, 2) \rightarrow \pi_{7}\left(M^{\bullet}\right)\right.$ fits into the following commutative diagram of exact sequences:

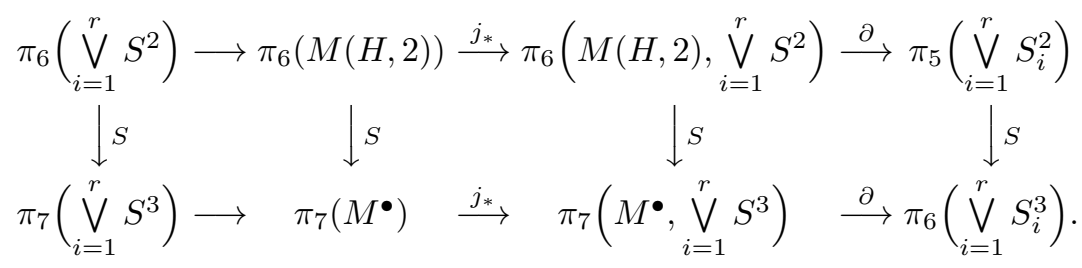

Let $X_{4, i}$ generate the homotopy group $\pi_{4}\left(S_{i}^{3} \cup_{k(i)} e_{i}^{4}, S^{3}\right) \cong \mathbb{Z}$, and let $\Psi$ generate $\pi_{7}\left(D^{4}, S^{3}\right) \cong \pi_{6}\left(S^{3}\right) \cong \mathbb{Z} / 12$. On classes of the form $X_{4, i} \circ \Psi$, the boundary map $\partial$ is given by multiplication by $k(i)$ : this is clear from the definition; see [29. Lemma 1]. An analogous statement holds for $X_{3, i}$ a generator of $\pi_{3}\left(S_{i}^{2} \cup_{k(i)}\right.$ $\left.e_{i}^{3}, S_{i}^{2}\right) \cong \mathbb{Z}$ and compositions $X_{3, i} \circ \Phi$ for $\Phi$ a generator of $\pi_{6}\left(D^{3}, S^{2}\right) \cong \pi_{5}\left(S^{2}\right) \cong$ $\mathbb{Z} / 2$. The stabilisation map $S: \pi_{5}\left(S^{2}\right) \rightarrow \pi_{6}\left(S^{3}\right)$ maps $\Phi$ to $6 \Psi$ by [31, (5.3)]. It follows for $i \leq s$ that the classes $X_{3, i} \circ \Phi$ and $X_{4, i} \circ(6 \Psi)$ are in the image of the maps $j_{*}$ and that $S\left(X_{3, i} \circ \Phi\right)=X_{4, i} \circ(6 \Psi)$. We choose $\varphi_{i} \in \pi_{6}(M(H, 2))$ such that

$$
j_{*}\left(\varphi_{i}\right)=X_{3, i} \circ \Phi
$$

and define

$$
\psi_{i}:=S\left(\varphi_{i}\right) \in \pi_{7}\left(M^{\bullet}\right)
$$

so that in particular $j_{*}\left(\psi_{i}\right)=X_{4, i} \circ(6 \Psi)$.

To see that the homotopy classes $\psi_{i}$ have the stated property, in particular that they are non-zero, we pass to stable homotopy groups: denoted $\pi_{*}^{S}$ with stabilisation $S: \pi_{*} \rightarrow \pi_{*}^{S}$. There is a commutative diagram

$$
\begin{array}{cc}
\pi_{7}^{S}\left(M^{\bullet}\right) \stackrel{j_{*}}{\longrightarrow} \pi_{7}^{S}\left(M^{\bullet}, \bigvee_{i=1}^{r} S^{3}\right) \\
\downarrow^{C_{*}} & \downarrow^{C_{*}} \\
\pi_{7}^{S}\left(S^{4}\right) \stackrel{\cong}{\longrightarrow} & \pi_{7}^{S}\left(S^{4}, *\right),
\end{array}
$$

where we also use $C$ to denote the corresponding map of pairs $\left(M^{\bullet}, \bigvee_{i=1}^{r} S^{3}\right) \rightarrow$ $\left(S^{4}, *\right)$ and $C_{*}$ the induced map on stable homotopy. By excision $j_{*}$ is an isomorphism and since $X_{4, i}$ stabilises to generate the group $\pi_{4}^{S}\left(S_{i}^{3} \cup_{k(i)} e_{i}^{4}, S_{i}^{3}\right) \cong \mathbb{Z}$ we quickly deduce that $C_{*} S\left(\psi_{i}\right)$ is the element of order two in $\pi_{7}^{S}\left(S_{i}^{4}\right)$. The stabilisation map $\pi_{6}\left(S^{3}\right) \rightarrow \pi_{3}^{S}$ is isomorphic to the inclusion $\mathbb{Z} / 12 \rightarrow \mathbb{Z} / 24$ and this completes the proof.

2.16. Definition. Using the homotopy equivalence $M^{\bullet} \simeq \bigvee_{i=1}^{r} M(\mathbb{Z} / k(i), 3)$ define generators $\left\{x_{1}, \ldots, x_{s}\right\}$ of $H^{3}(M ; \mathbb{Z} / 2)$, where each $x_{i}$ is the pullback of the generator of $H^{3}(M(\mathbb{Z} / k(i)) ; \mathbb{Z} / 2)=\mathbb{Z} / 2$. Given $x \in H^{3}(M ; \mathbb{Z} / 2)$ write $x=\sum_{i=1}^{s} \epsilon_{i} x_{i}$ where $\epsilon_{i}=0$ or 1 and define

$$
p(x):=p\left(\sum_{i=1}^{s} \epsilon_{i} \psi_{i}\right)
$$

with $\psi_{i} \in \pi_{7}\left(M^{\bullet}\right)$ defined as in (2.8). 
To complete the proof of part (10) of Lemma 2.14 it remains to show that we have $\eta^{P L}(p(x))=x \in H^{3}(M ; \mathbb{Z} / 2) \subset[M, G / P L]$. To do this we shall apply the results of [28, which are stated in the setting of tangential surgery for manifolds with boundary. We briefly recall the salient features of this setting without giving all the details: for these we refer the reader to [28, §2].

Recall that the manifold $M$ decomposes as $M=M^{\bullet} \cup_{S^{6}} D^{7}$. The structure set of $M^{\bullet}$, not relative to the boundary, consists of equivalence classes of homotopy equivalences of pairs

$$
h:(N, \partial N) \rightarrow\left(M^{\bullet}, S^{6}\right),
$$

where $N$ is a compact 7-manifold with boundary. We may assume that any element $[h] \in \mathcal{S}^{C a t}(M)$ has a representative $h: N \simeq M$, where $\left.h\right|_{D^{7}}$ is a diffeomorphism and $h\left(N^{\bullet}\right)=M^{\bullet}$ and so there is a well-defined map

$$
F^{\bullet}: \mathcal{S}^{C a t}(M) \rightarrow \mathcal{S}^{C a t}\left(M^{\bullet}\right), \quad[h] \mapsto\left[\left.h\right|_{N} \bullet\right] .
$$

By the $(\pi-\pi)$-Theorem [34, Theorem 3.3], the normal invariant defines a bijection $\eta^{\text {Cat }}: \mathcal{S}^{\text {Cat }}\left(M^{\bullet}\right) \rightarrow\left[M^{\bullet}, G / C a t\right]$ and so by (2.6) and the proof of Lemma 2.12 we deduce that there are bijections

$$
\mathcal{S}^{C a t}(M) \stackrel{F^{\bullet}}{\longrightarrow} \mathcal{S}^{C a t}\left(M^{\bullet}\right) \stackrel{\eta^{C a t}}{\longrightarrow}\left[M^{\bullet}, G / C a t\right] .
$$

Writing $p\left(\psi_{i}\right):\left(M^{\bullet}, S^{6}\right) \rightarrow\left(M^{\bullet}, S^{6}\right)$ also for the pinch map of the pair $\left(M^{\bullet}, S^{6}\right)$ corresponding to the homotopy equivalence $p(\psi): M \simeq M$, we choose a bundle isomorphism $b\left(\psi_{i}\right): \nu_{M} \bullet \nu_{M} \bullet$ covering $p\left(\psi_{i}\right)$. The pair $\left(p\left(\psi_{i}\right), b\left(\psi_{i}\right)\right)$ defines an element in the tangential structure set of $M^{\bullet}, \mathcal{S}^{C a t, t}\left(M^{\bullet}\right)$. There are forgetful maps from tangential surgery to usual surgery which fit into the following commutative square (see [28, (2.4)]):

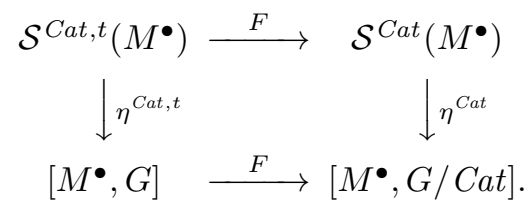

Here $\eta^{C a t, t}$ is the normal invariant of tangential surgery and the lower forgetful map induced by the projection $G \rightarrow C /$ Cat. Now by [1] the natural map $O \rightarrow P L$ is a 6 -equivalence and so the exact sequence

$$
\pi_{4}(G / P L) \rightarrow \pi_{3}(P L) \rightarrow \pi_{3}(G)
$$

is isomorphic to the same sequence with $P L$ replaced by $O$. By [1, Example 7.17] the homomorphism $\pi_{3}(O) \rightarrow \pi_{3}(G)$ is isomorphic to the surjection $\mathbb{Z} \rightarrow \mathbb{Z} / 24$ and by [31. Proposition 5,18] $\pi_{4}(G)=0$. It follows that the sequence (2.9) above is isomorphic to the short exact sequence

$$
\mathbb{Z} \stackrel{\times 24}{\longrightarrow} \mathbb{Z} \rightarrow \mathbb{Z} / 24
$$

A further elementary obstruction theory argument shows for $C a t=P L$ that the homomorphism $\left[M^{\bullet}, G\right] \rightarrow\left[M^{\bullet}, G / P L\right]$ is isomorphic to the boundary map

$$
\beta_{24}: H^{3}(M ; \mathbb{Z} / 24) \rightarrow H^{4}(M ; \mathbb{Z})
$$

in the cohomology Bockstein sequence defined by (2.10). It is then a simple matter to check that these facts and the following lemma complete the proof of part (1) of Lemma 2.14. 
2.17. Lemma. The tangential normal invariant of $\left(p\left(\psi_{i}\right), b\left(\psi_{i}\right)\right)$ is independent of $b\left(\psi_{i}\right)$ and given by the equation

$$
\eta^{P L, t}\left(p\left(\psi_{i}\right), b\left(\psi_{i}\right)\right)=\iota\left(x_{i}\right) \in H^{3}(M ; \mathbb{Z} / 24) \cong\left[M^{\bullet}, G\right],
$$

where $x_{i}$ is as in Definition 2.16 and $\iota: H^{3}(M ; \mathbb{Z} / 2) \rightarrow H^{3}(M ; \mathbb{Z} / 24)$ is induced by the inclusion of coefficients $\mathbb{Z} / 2 \hookrightarrow \mathbb{Z} / 24$.

Proof. The tangential normal invariant of $(p, b):=\left(p\left(\psi_{i}\right), b\left(\psi_{i}\right)\right)$ is computed as follows: first use the Pontryagin-Thom isomorphism to obtain an element in the stable homotopy group of the pair $\left(T\left(\nu_{M} \bullet\right), T\left(\nu_{M} \bullet \mid *\right)\right)$, where $T(\xi)$ denotes the Thom space of a vector bundle which we assume has rank $k>8$ and $* \in \partial M^{\bullet}$ is a base-point in the boundary of $M^{\bullet}$. We write:

$$
P T(p, b) \in \pi_{7+k}\left(T\left(\nu_{M} \bullet\right), T\left(\nu_{M} \bullet \mid *\right)\right) .
$$

Next one applies Spanier-Whitehead duality

$$
D: \pi_{7+k}\left(T\left(\nu_{M} \bullet\right), T\left(\nu_{M} \bullet *\right)\right) \longrightarrow[M, G]
$$

and defines $\eta^{C a t, t}(p, b):=D(P T(p, b))$.

Now $M^{\bullet}=\Sigma M(H, 2)$ is a suspension and so $T\left(\nu_{M} \bullet\right) / T\left(\nu_{M} \bullet \mid *\right) \simeq \Sigma^{k} M^{\bullet}$ (see the remark at the bottom of [28, p. 469]). Moreover, the pinch map $p\left(\psi_{i}\right)$ is defined using a suspension $\psi_{i}=S\left(\varphi_{i}\right), \varphi_{i} \in \pi_{6}(M(H, 2))$. It follows by [28, Theorem 4.7 and Lemma 4.8] that

$$
\eta^{P L, t}\left(p\left(\psi_{i}\right), b\left(\psi_{i}\right)\right)=D\left(S\left(\psi_{i}\right)\right)
$$

where $S\left(\psi_{i}\right) \in \pi_{7}^{S}\left(M^{\bullet}\right)$ is the stabilisation of $\psi_{i}$. But by the proof of Lemma 2.15 we know that $C_{*} S\left(\psi_{i}\right) \in \pi_{7}^{S}\left(\bigvee_{i=1}^{r}\left(S_{i}^{4}\right)\right)$ is precisely the element of order two in the summand $\pi_{7}^{S}\left(S_{i}^{4}\right) \cong \mathbb{Z} / 24$. Applying Spanier-Whitehead duality to this statement completes the proof.

We now prove part (2) of Lemma 2.14 If $x=0$, then $p(x) \simeq$ Id and the statement is obvious. Assume then that $x \neq 0 \in H^{3}(M ; \mathbb{Z} / 2)$. By choosing an appropriate set of generators for $H_{3}(M)$ we may assume that $x=x_{1}$ in the notation of Definition 2.16. Let $f: M \rightarrow B S^{3}$ classify $E \in \operatorname{Bun}(M)$. As $p\left(x_{1}\right)$ is the pinch map on $\psi_{1} \in \pi_{7}\left(M^{\bullet}\right)$ the induced map $p\left(x_{1}\right)^{*}$ of $\operatorname{Bun}(M)$ is given by

$$
p\left(x_{1}\right)^{*} E=E \sharp F_{E},
$$

where $F_{E} \in \operatorname{Bun}\left(S^{7}\right)$ is classified by the composition

$$
\left.f\right|_{M} \bullet \psi_{1}: S^{7} \longrightarrow M^{\bullet} \longrightarrow B S^{3} .
$$

But $B S^{3}$ is 3-connected and so $\left.f\right|_{M}$. factors through the collapse map $C$ of (2.7):

$$
\left.f\right|_{M} \bullet=\bar{f} \circ C: M^{\bullet} \longrightarrow \bigvee_{i=1}^{r} S_{i}^{4} \longrightarrow B S^{3} .
$$

By definition $\psi_{1}: S^{7} \rightarrow M^{\bullet}$ is such that $C_{*}\left(\psi_{1}\right)=(0,6) \in \pi_{7}\left(S_{1}^{4}\right) \cong \mathbb{Z} \oplus \mathbb{Z}_{12}$. Recall that $H \rightarrow \mathbb{H} P^{\infty}$ is the tautological bundle and so $F_{E}=\left(\left.f\right|_{M} \bullet \circ \psi_{1}\right)^{*} H$ is determined by $c_{2}(E)$ as follows:

$$
F_{E}= \begin{cases}6 \in \pi_{7}\left(B S^{3}\right) \cong \mathbb{Z} / 12, & \text { if } c_{2}(E)\left[S_{1}^{4}\right] \text { is odd }, \\ 0 \in \pi_{7}\left(B S^{3}\right) \cong \mathbb{Z} / 12, & \text { if } c_{2}(E)\left[S_{1}^{4}\right] \text { is even } .\end{cases}
$$


Applying Theorem 2.4 (11) and Proposition 1.5 (2) to (2.11) and (2.12) above we see that

$$
t_{M}\left(p\left(x_{1}\right)^{*} E\right)-t_{M}(E)=t_{S^{7}}\left(F_{E}\right)=\left(x_{1} \smile c_{2}(E)\right)[M]_{2} \in \mathbb{Z} / 2 \subset \mathbb{Q} / \mathbb{Z} .
$$

2.c. Remarks on simply connected 7-manifolds. In this subsection we make some remarks about the role of the $t$-invariant in the classification of simply connected spin 7-manifolds.

2.18. Remark. The invariant $t_{M}$ is related to the Kreck-Stolz invariants $s_{2}$ and $s_{3}$ of 25. To begin let $M$ be a closed spin $(4 k-1)$-manifold as in Definition 1.4, so $H^{3}(M ; \mathbb{Q})=0$. Let $L \rightarrow M$ be a Hermitian line bundle with $c_{1}(L)=a \in H^{2}(M)$ such that $c_{1}(L)^{2} \in H^{4}(M)$ is a torsion class. In analogy with (1.3), we define a characteristic class $\mathrm{ch}^{\prime}$ of complex line bundles such that

$$
\operatorname{ch}(L)=1+c_{1}(L)+c_{1}(L)^{2} \operatorname{ch}^{\prime}(L) .
$$

Assume that $L$ extends to $\bar{L} \rightarrow W$, where $W$ is a compact spin manifold with $M=$ $\partial W$. The class $c_{1}(\bar{L})^{2} \in H^{4}(W)$ lifts uniquely to $\bar{v} \in H^{4}(W, M ; \mathbb{Q})$, so we can define

$$
s_{M}(a):=\left(\widehat{A}(T W) \operatorname{ch}^{\prime}(\bar{L}) \bar{v}\right)[W, M] \in \mathbb{Q} / \mathbb{Z}
$$

independent of the choices of $W$ and $\bar{L}$. Because $W$ is $4 k$-dimensional, we have that $s_{M}(a)=s_{M}(-a)$ for all $a \in H^{2}(M)$.

A Hermitian metric on $L$ identifies the dual bundle $L^{*}$ with the complex conjugate of $L$, and this identification is parallel with respect to compatible Hermitian connections. Thus the bundle

$$
E:=L \oplus L^{*}
$$

carries a natural quaternionic structure with $c_{2}(E)=-c_{1}(L)^{2}$. In particular, we have

$$
\operatorname{ch}(E)=\operatorname{ch}(L)+\operatorname{ch}\left(L^{*}\right) \quad \text { and } \quad \operatorname{ch}^{\prime}(E)=\operatorname{ch}^{\prime}(L)+\operatorname{ch}^{\prime}\left(L^{*}\right)
$$

and $\bar{v}=-\bar{c}_{2}(E)$. Thus $a_{k+1} t_{M}(E)=s_{M}(a)+s_{M}(-a)=2 s_{M}(a) \in \mathbb{Q} / a_{k+1} \mathbb{Z}$, or equivalently

$$
t_{M}(E)=a_{k} s_{M}(a) .
$$

Hence the $t$-invariant generalises the $s$-invariant if $k$ is even. There is an analogous argument comparing the intrinsic definitions of $s_{M}$ and $t_{M}$.

In [25], Kreck and Stolz consider the case $k=2$, so that $M$ is 7-dimensional, and $H^{4}(M)$ is a torsion group by Poincaré duality. They also assume that $M$ is simply connected, that $H^{2}(M) \cong \mathbb{Z}$ with an arbitrary generator $z, H^{3}(M) \cong$ 0 , and that $z^{2}$ generates $H^{4}(M)$. They consider the invariants $s_{2}(M):=s_{M}(z)$ and $s_{3}(M):=s_{M}(2 z)$. In particular, $t_{M}$ generalises $s_{2}$ and $s_{3}$. Note that Kreck and Stolz also define invariants $s_{2}, s_{3}$ for similar $M$ as above that are not spin. These invariants are not covered by the $t$-invariant.

2.19. Remark. Let us now consider simply connected closed spin 7-manifolds $M$ with $H^{3}(M)=0$. Then $H^{2}(M) \cong \mathbb{Z}^{r}$ for some $r$, and $H^{4}(M)$ is finite by the universal coefficient theorem and Poincaré duality.

Expanding on [24, Theorem 6], Hepworth [23, Thereom 2.2.9] gave a classification theorem under the additional assumption that $H^{4}(M)$ is generated by $p_{M}=$ $\frac{p_{1}}{2}(T M)$ and cup products of elements of $H^{2}(M)$. Apart from the Eells-Kuiper invariant, the linking form, and the triple Massey products, Hepworth chooses a $\mathbb{Z}$ basis of $H^{2}(M)$ and defines five more families of invariants, $\sigma_{i}, \sigma_{i j}, \tau_{i}, \tau_{i j}$, and $\tau_{i j, k}$ 
for $i, j, k \in\{1, \ldots, r\}$, using a compact spin manifold $W$ with $\partial W=M$ such that the inclusion $M \rightarrow W$ induces an isomorphism $H^{2}(W) \cong H^{2}(M)$. Together, these invariants give a complete set of diffeomorphism invariants.

One can show that the linking form and the $\sigma$ - and $\tau$-invariants can be expressed in terms of the function $s_{M}$ of Remark 2.18 and some Massey products. As above, we may use $t_{M}\left(L \oplus L^{*}\right)$ instead of $s_{M}(L)$.

If we now drop the assumption that $H^{4}(M)$ is generated by $p_{M}$ and products of elements of $H^{2}(M)$, then, as in the highly connected case, the invariant $t_{M}$ contains potentially more information than $s_{M}$ because one can define $t_{M}(E)$ even if $c_{2}(E)$ is not in the span of $H^{2}(M) \smile H^{2}(M)$. It therefore seems reasonable to hope that simply connected spin 7-manifolds $M$ with $H^{3}(M)=0$ can be classified via their Eells-Kuiper invariants, their Massey product structure, and their $t$-invariants.

\section{BUNDLES OVER $\mathbb{H} P^{k}$}

In this section we investigate the map $c_{2}$ : $\operatorname{Bun}\left(\mathbb{H} P^{k}\right) \rightarrow H^{4}\left(\mathbb{H} P^{k}\right)$ using the $t$-invariant. Determining the image of $c_{2}$ is a difficult problem already investigated in [18, 20 and elsewhere. One calls an integer $c k$-realisable if there is a bundle $E \in$ $\operatorname{Bun}\left(\mathbb{H} P^{k}\right)$ such that

$$
c_{2}(E)=c \cdot c_{2}(H),
$$

where we recall that $H$ is the tautological bundle over $\mathbb{H} P^{k}$. Below we use the $t$-invariant to give a new proof of the necessary criteria of [18 for $k$-realisability.

Consider the Hopf fibration $p_{k}: S^{4 k-1} \rightarrow \mathbb{H} P^{k-1}$ and let $\bar{p}_{k}: W_{k} \rightarrow \mathbb{H} P^{k-1}$ denote the corresponding $D^{4}$-bundle with boundary $S^{4 k-1}$. Then

$$
\mathbb{H} P^{k}=W_{k} \cup_{S^{4 k-1}} D^{4 k} .
$$

One may therefore consider the problem of building bundles over $\mathbb{H} P^{k}$ inductively: assume that $E_{k-1}$ is a quaternionic line bundle on $\mathbb{H} P^{k-1}$; then the pullback $\bar{p}_{k}^{*} E_{k-1}$ on $W$ extends to $E_{k} \rightarrow \mathbb{H} P^{k}$ if and only if its restriction $\left.\bar{p}_{k}^{*} E_{k-1}\right|_{S^{4 k-1}}=p_{k}^{*} E_{k-1}$ to the boundary is trivial. If $p_{k}^{*} E_{k-1}$ is trivial, then the group $\pi_{4 k-1}\left(S^{3}\right)$ acts transitively on the set of possible extensions of $E_{k-1}$ to $\mathbb{H} P^{k}$. The situation is summarised in the following commutative diagram:

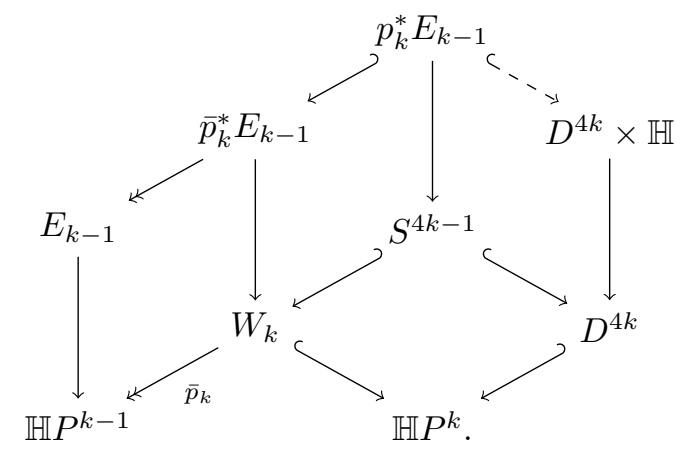

For the calculations below we now fix $a:=-c_{2}(H)$ as a generator of $H^{4}\left(\mathbb{H} P^{k}\right)$. 
3.1. Theorem. If $k \geq 2$ and there exists a quaternionic line bundle $E_{c}$ on $\mathbb{H} P^{k-1}$ with $c_{2}\left(E_{c}\right)=-c \cdot a$ in $H^{4}\left(\mathbb{H} P^{k-1}\right) \cong \mathbb{Z} a$, then

$$
t_{S^{4 k-1}}\left(p_{k}^{*} E_{c}\right)=\frac{a_{k}}{(2 k) !} \prod_{j=0}^{k-1}\left(c-j^{2}\right) \in \mathbb{Q} / \mathbb{Z} .
$$

We shall prove Theorem 3.1 at the end of the section.

3.2. Corollary ([18, Theorem 1.1). If an integer $c$ is $k$-realisable, then

$$
\frac{a_{j}}{(2 j) !} \prod_{i=0}^{j-1}\left(c-i^{2}\right)=0 \in \mathbb{Q} / \mathbb{Z} \quad \text { for all } 2 \leq j \leq k .
$$

Proof. Let $E_{c} \in \operatorname{Bun}\left(\mathbb{H} P^{k}\right)$ be a bundle with $c_{2}\left(E_{c}\right)=c \cdot c_{2}(\tau)=-c \cdot a$. Then for all $2 \leq j \leq k$, the bundle $E_{c}^{j}:=p_{j}^{*}\left(\left.E_{c}\right|_{H P^{j-1}}\right)=\left.E_{c}\right|_{S^{4 j-1}}$ is trivial and in particular $t_{S^{4 j-1}}\left(E_{c}^{j}\right)=0$. Now apply Theorem 3.1 .

3.3. Remark. Feder and Gitler proved that the conditions of Corollary 3.2 are necessary using different methods in 18 . They also gave a proof that the conditions are sufficient if $k=\infty 11$ where they are satisfied if and only if $c$ is an odd square or zero. Feder and Gitler strongly suggested that their conditions are also sufficient for finite $k$, but so far, this has been proved only for $k=2,3,4,5$ (see [20, §2] for more details on this issue). Moreover, the bundle $E_{c}$ is not unique in general; however, Gonçalves and Spreafico [20, Theorem 3] proved that the number of nonisomorphic bundles with the same second Chern class is finite and depends only on $k$ and the parity of $c$.

3.4. Remark. Theorem 3.1 and Theorem 1.12 shed some light on the Feder-Gitler conditions: the a priori obstruction to extending a bundle $E_{c} \rightarrow \mathbb{H} P^{j-1}$ to $\mathbb{H} P^{j}$ is the bundle $p_{j}^{*} E_{c} \in \operatorname{Bun}\left(S^{4 j-1}\right) \cong \pi_{4 j-2}\left(S^{3}\right)$. We see that the claim that the Feder-Gitler conditions are sufficient for $c$ to be $k$-realisable is equivalent to the following claim: For each $2 \leq j \leq k$ such that $c$ is $(j-1)$-realisable, the set

$$
t_{S^{4 j-1}}^{-1}(0) \cap p_{j}^{*}\left(c_{2}^{-1}\left(c \cdot c_{2}(H)\right)\right) \subset \operatorname{Bun}\left(S^{4 j-1}\right)
$$

contains the trivial bundle $S^{4 j-1} \times \mathbb{H}$. By Theorem 3.1 and Corollary 1.13 this is true if $k=2$ or 3 but becomes surprising if $k>3$. By Proposition 1.5 the $t$ invariant measures only minus the $e$-invariant of the stabilisation $S\left(p_{j}^{*} E_{c}\right) \in \pi_{4 k-5}^{S}$ and for $k>3$ the stabilisation homomorphism $S: \pi_{4 k-2}\left(S^{3}\right) \rightarrow \pi_{4 k-5}^{S}$ and the $e$-invariant $e: \pi_{4 k-5}^{S} \rightarrow \mathbb{Q} / \mathbb{Z}$ both typically have non-trivial kernels.

We next discuss Theorem 3.1 for $k=2,3$ and 4 .

3.5. Example. For $k=2$, we recover a special case of Proposition 2.6. In particular, there exist quaternionic line bundles $E_{c}$ on $\mathbb{H} P^{1}=S^{4}$ with $c_{2}\left(E_{c}\right)=-c \cdot a$ for all $c \in \mathbb{Z}$, and we have

$$
t_{S^{7}}\left(p_{2}^{*} E_{c}\right)=\frac{c(c-1)}{24} \in \frac{1}{12} \mathbb{Z} / \mathbb{Z} .
$$

This confirms Theorem 2.4 (1) stating that $t_{S^{7}}$ is injective. In fact, $\operatorname{Bun}\left(S^{7}\right) \cong$ $\pi_{6}\left(S^{3}\right) \cong \mathbb{Z} / 12$ is a group under the connected sum operation, and $t: \operatorname{Bun}\left(S^{7}\right) \rightarrow$ $\mathbb{Z} / 12$ is an isomorphism.

\footnotetext{
${ }^{1}$ In [18], Feder and Gitler quote Sullivan who credits this statement to unpublished work of I. Bernstein, R. Stong, L. Smith and G. Cooke.
} 
3.6. Example. A bundle $p_{2}^{*} E_{c}$ on $S^{7}$ is trivial if and only if $c(c-1) \equiv 0 \bmod 24$. This is the case if and only if

$$
c \equiv 0,1 \bmod 8 \quad \text { and } \quad c \equiv 0,1 \bmod 3 .
$$

Because $\pi_{7}\left(S^{3}\right) \cong \mathbb{Z}_{2}$, there exist at most two extensions of $E_{c}$ to $\mathbb{H} P^{2}$ up to isomorphism. We pick one for each $c$ and still denote it by $E_{c}$.

By Theorem 3.1.

$$
t_{S^{11}}\left(p_{3}^{*} E_{c}\right)=\frac{2 c(c-1)(c-4)}{720}=\frac{c(c-1)}{24} \cdot \frac{c-4}{15} \in \frac{1}{15} \mathbb{Z} / \mathbb{Z},
$$

because the first factor is always an integer. Because $E_{c}$ exists on $\mathbb{H} P^{2}$ for $c=33$ and

$$
t_{S^{11}}\left(p_{3}^{*} E_{33}\right)=\frac{1}{15},
$$

we conclude from Proposition [1.5 (2) that the $t$-invariant again gives an isomorphism

$$
t_{S^{11}}: \operatorname{Bun}\left(S^{11}\right) \cong \pi_{10}\left(S^{3}\right) \cong \mathbb{Z} / 15 \longrightarrow \mathbb{Z} / 15
$$

This confirms Corollary 1.13 stating that $t_{S^{11}}$ is injective. It also shows that the possible extensions of $E_{c}$ from $\mathbb{H} P^{1}$ to $\mathbb{H} P^{2}$ become isomorphic on $S^{11}$.

3.7. Example. A bundle $p_{3}^{*} E_{c}$ exists and is trivial on $S^{11}$ if and only if

$$
c \equiv 0,1 \bmod 8, \quad c \equiv 0,1,4,7 \bmod 9, \quad \text { and } \quad c \equiv 0,1,4 \bmod 5 \text {. }
$$

In this case, it extends to $\mathbb{H} P^{3}$. Note that because $\pi_{11}\left(S^{3}\right) \cong \mathbb{Z} / 2$ and because there were possibly two non-isomorphic bundles $E_{c}$ on $\mathbb{H} P^{2}$ to start with, there can be up to four quaternionic line bundles on $\mathbb{H} P^{3}$ with second Chern class $-c \cdot a \in H^{4}\left(\mathbb{H} P^{3}\right)$. We pick one and again denote it by $E_{c}$.

By Theorem 3.1,

$$
t_{S^{15}}\left(p_{4}^{*} E_{c}\right)=\frac{c(c-1)(c-4)(c-9)}{2^{7} \cdot 3^{2} \cdot 5 \cdot 7} \in \frac{1}{28} \mathbb{Z} / \mathbb{Z},
$$

because the numerator is always divisible by $2^{5} \cdot 3^{2} \cdot 5$ for all $c \in \mathbb{Z}$ that can occur. For $c=40$, we have

which generates $\frac{1}{28} \mathbb{Z} / \mathbb{Z}$.

$$
t_{S^{15}}\left(p_{4}^{*} E_{40}\right)=\frac{5}{28},
$$

Now by [31, (7.14), Theorem 13.9, (13.6)'] we see that the stabilisation homomorphism $S: \pi_{14}\left(S^{3}\right) \rightarrow \pi_{11}^{S}$ is isomorphic to the homomorphism

$$
\mathbb{Z} / 84 \oplus \mathbb{Z} / 2 \oplus \mathbb{Z} / 2 \stackrel{(\times 6,0,0)}{\longrightarrow} \mathbb{Z} / 504,
$$

and by [1] the $e$-invariant $e: \pi_{11}^{S} \rightarrow \mathbb{Q} / \mathbb{Z}$ is injective. Hence, by Theorem 1.12, the $t$-invariant defines an injective homomorphism on $\mathbb{Z} / 84 \oplus 0 \oplus 0 \subset \pi_{14}\left(S^{3}\right)$. We have seen above that $t_{S^{15}}\left(p^{*} E_{c}\right) \in \frac{1}{28} \mathbb{Z} / \mathbb{Z}$, which implies that the 3 -torsion component in $\operatorname{Bun}\left(S^{15}\right)$ cannot be generated by bundles of the type $p_{4}^{*} E_{c}$.

Proof of Theorem 3.1. We compute $t_{S^{4 k-1}}$ using the zero bordism $W_{k}$ as in Definition (1.6). Using the homotopy equivalence $\bar{p}_{k}: W_{k} \rightarrow \mathbb{H} P^{k}$ to identify $H^{4}\left(W_{k}\right)=$ $H^{4}\left(\mathbb{H} P^{k-1}\right)$ and the Thom isomorphism, we know that the generator $a \in H^{4}\left(W_{k}\right)$ lifts to a generator of $H^{4}\left(W_{k}, S^{4 k-1}\right) \cong a \mathbb{Z}[a] / a^{k+1} \mathbb{Z}[a]$. In particular,

$$
\bar{c}_{2}\left(E_{c}\right) \operatorname{ch}^{\prime}\left(E_{c}\right)=2-2 \cosh (\sqrt{c a}) \in H^{\bullet}\left(W_{k}, S^{4 k-1} ; \mathbb{Q}\right) \cong a \mathbb{Q}[a] / a^{k+1} \mathbb{Q}[a] .
$$


A quaternionic Hermitian metric $g$ identifies $\bar{H}$ with $H^{*}$ by sending an element $h \in H$ to $g(h, \cdot)$. Note that $\bar{H}$ has the same total space as $H$, but $q \in \mathbb{H}$ acts by multiplication with $\bar{q}$. Hence, if $\mathbb{H}$ acts on $H$ from the right, then the action on $\bar{H}$ is a left action, and we can regard the tensor product $\operatorname{End}_{\mathbb{H}} H \cong H \otimes_{\mathbb{H}} H^{*} \cong H \otimes_{\mathbb{H}} \bar{H}$ as a real vector bundle.

The tangent bundle of $\mathbb{H} P^{k}$ is a real vector bundle satisfying

$$
T \mathbb{H} P^{k} \oplus \operatorname{End}_{\mathbb{H}} H \cong H_{\mathbb{R}}^{\oplus(k+1)},
$$

where we write $H_{\mathbb{R}}$ for $H$ regarded as a real vector bundle. We will now compute the characteristic classes of $\operatorname{End}_{\mathbb{H}} H$ and $H_{\mathbb{R}}$.

We can regard $H$ as a complex vector bundle. Note that multiplication with $j$ induces an isomorphism of $H$ and $\bar{H}$ as complex vector bundles, where $i, j, k$ are the basic imaginaries of $\mathbb{H}$. In particular, $c_{1}(H)=0$. Writing $I$ for the imaginary of $\mathbb{C}$, we see that there is a natural isomorphism $H_{\mathbb{R}} \otimes_{\mathbb{R}} \mathbb{C} \cong H \oplus H$ given by

$$
q \otimes 1+r \otimes I \longmapsto(q+r i, r-q i) .
$$

Hence, the total Pontryagin class of $H_{\mathbb{R}}$ is given by

$$
p\left(H_{\mathbb{R}}\right)=1-c_{2}\left(H_{\mathbb{R}} \otimes_{\mathbb{R}} C\right)+c_{4}\left(H_{\mathbb{R}} \otimes_{\mathbb{R}} \mathbb{C}\right)=\left(1-c_{2}(H)\right)^{2} .
$$

Turning to $\operatorname{End}_{\mathbb{H}} H$, one can conclude from Schur's Lemma and the representation theory of $S O(4)=\left(S^{3} \times S^{3}\right) /(\mathbb{Z} / 2)$ that the complex bundles

$$
\left(\operatorname{End}_{\mathbb{H}} H\right) \otimes_{\mathbb{R}} \mathbb{C} \cong\left(H \otimes_{\mathbb{H}} \bar{H}\right) \otimes_{\mathbb{R}} \mathbb{C} \quad \text { and } H \otimes_{\mathbb{C}} H
$$

are isomorphic. An explicit isomorphism can be given by

$$
(q \otimes w) \otimes z \longmapsto q z \otimes w j-q j z \otimes w=q \otimes w j z-q j \otimes w z
$$

for all points $p \in \mathbb{H} P^{k}$, all $q, w \in H_{p}$ and all $z \in \mathbb{C}$. To check that this is welldefined, regard $h=x+j y \in \mathbb{H}$ with $x, y \in \mathbb{C}$. Then $(q h \otimes w) \otimes z=(q \otimes w \bar{h}) \otimes z$ is mapped to

$$
\begin{aligned}
q h \otimes w j z-q h j \otimes w z & =q(x+j y) \otimes w j z-q(x+j y) j \otimes w z \\
& =q \otimes w j x z+q j \otimes w j y z-q j \otimes w \bar{x} z+q \otimes w \bar{y} z \\
& =q \otimes w(\bar{x}-\bar{y} j) j z-q j \otimes w(\bar{x}-\bar{y} j) z \\
& =q \otimes w \bar{h} j z-q j \otimes w \bar{h} z .
\end{aligned}
$$

We can now compute the characteristic classes of $\operatorname{End}_{\mathbb{H}} H$. By the splitting principle [3, Corollary 2.7.11], it suffices to treat the case that $H \cong L \oplus L^{\prime}$ is isomorphic to the sum of two complex line bundles. Because $c_{1}(H)=0$, we conclude that $L^{\prime} \cong \bar{L}$, so that

$$
1-a=c(H)=\left(1+c_{1}(L)\right) \cdot\left(1-c_{1}(L)\right)=1-c_{1}(L)^{2}
$$

by multiplicativity of the total Chern class. Similarly,

$$
\begin{aligned}
c\left(\left(\operatorname{End}_{\mathbb{H}} H\right) \otimes_{\mathbb{R}} \mathbb{C}\right) & =c\left(L^{\otimes 2} \oplus \bar{L}^{\otimes 2} \oplus \varepsilon \oplus \varepsilon\right) \\
& =\left(1+2 c_{1}(L)\right) \cdot\left(1-2 c_{1}(L)\right) \cdot 1^{2}=1-4 c_{1}(L)^{2}=1-4 a .
\end{aligned}
$$

In particular,

$$
\begin{aligned}
p_{1}\left(\operatorname{End}_{\mathbb{H}} H\right) & =-c_{2}\left(\left(\operatorname{End}_{\mathbb{H}} H\right) \otimes_{\mathbb{R}} \mathbb{C}\right)=4 a \\
\text { and } \quad p_{2}\left(\operatorname{End}_{\mathbb{H}} H\right) & =c_{4}\left(\left(\operatorname{End}_{\mathbb{H}} H\right) \otimes_{\mathbb{R}} \mathbb{C}\right)=0
\end{aligned}
$$


Since the identity endomorphism gives a nowhere vanishing section of $\operatorname{End}_{\mathbb{H}} H$ we deduce that

$$
e\left(\operatorname{End}_{\mathbb{H}} H\right)=0 .
$$

From (3.2) and multiplicativity of the $\hat{A}$-class, we deduce that

$$
\begin{aligned}
\widehat{A}\left(T \mathbb{H} P^{k}\right) & =\widehat{A}(H)^{k+1} \smile \widehat{A}\left(\operatorname{End}_{\mathbb{H}} H\right)^{-1} \\
& =\left(\frac{\sqrt{a} / 2}{\sinh (\sqrt{a} / 2)}\right)^{2 k+2}\left(\frac{\sqrt{a}}{\sinh \sqrt{a}}\right)^{-1} \\
& =\left(\frac{\sqrt{a} / 2}{\sinh (\sqrt{a} / 2)}\right)^{2 k+1} \cosh (\sqrt{a} / 2) \\
& \in H^{\bullet}\left(\mathbb{H} P^{k} ; \mathbb{Q}\right) \cong \mathbb{Q}[a] / a^{k+1} \mathbb{Q}[a],
\end{aligned}
$$

and we know that $\widehat{A}\left(T W_{k}\right)=\left.\widehat{A}\left(T \mathbb{H} P^{k}\right)\right|_{W_{k}}$.

Let $\gamma$ be a sufficiently small contour around 0 in $\mathbb{C}$. Then $\gamma^{2}$ goes around 0 twice. Note that $a_{k} a_{k+1}=2$ for all $k$. From the formula for $\widehat{A}\left(T \mathbb{H} P^{k}\right)$ above and using the residue theorem and the fact that $a^{k}\left[W_{k}, S^{4 k-1}\right]=1$, we compute

$$
\begin{aligned}
t_{S^{4 k-1}}\left(p_{k}^{*} E_{c}\right) & =-\frac{1}{a_{k+1}}\left(\widehat{A}\left(T W_{k}\right) \bar{c}_{2}\left(\bar{p}_{k}^{*} E_{c}\right) \operatorname{ch}^{\prime}\left(\bar{p}_{k}^{*} E_{c}\right)\right)\left[W_{k}, S^{4 k-1}\right] \\
& =\frac{a_{k}}{2 \pi i} \int_{\gamma} \frac{\cosh (\sqrt{a} / 2)(\cosh (\sqrt{a c})-1)}{2^{2 k+1} \sqrt{a} \sinh (\sqrt{a} / 2)^{2 k+1}} d a \\
& =\frac{a_{k}}{2 \pi i} \int_{\gamma^{2}} \frac{\cosh (\sqrt{a} / 2)(\cosh (\sqrt{a c})-1)}{2^{2 k+2} \sqrt{a} \sinh (\sqrt{a} / 2)^{2 k+1}} d a \\
& =\frac{a_{k}}{2 \pi i} \int_{\gamma} \frac{\cosh \left(2 \sqrt{c} \cdot \operatorname{ar} \sinh \frac{z}{2}\right)-1}{z^{2 k+1}} d z \in \mathbb{Q} / \mathbb{Z} .
\end{aligned}
$$

In the last step, we have substituted $z$ for $2 \sinh (\sqrt{a} / 2)$. Note that the contour for $z$ closes only after $a$ has completed two cycles around the origin.

Writing cosh and sinh as linear combinations of exponential functions, one sees that there exist even polynomials $P_{n}$ of degree $2 n$ such that

$$
\cosh (2 n x)=P_{n}(\sinh x)=\sum_{k=0}^{n} p_{n, k} \sinh ^{2 k} x,
$$

and $p_{n, n}=2^{2 n-1}$. On the other hand, by the residue theorem,

$$
\bar{t}_{k}(y):=\frac{1}{2 \pi i} \int_{\gamma} \frac{\cosh \left(2 y \cdot \operatorname{ar} \sinh \frac{z}{2}\right)-1}{z^{2 k+1}} d z
$$

is an even polynomial of degree at most $2 k$ in $y$ such that $\bar{t}_{k}(n)=2^{-2 k} p_{n, k}$. Because $p_{n, k}=0$ if $n<k$, we conclude that $\bar{t}_{k}$ vanishes at $n=1-k, \ldots, k-1$, and that $\bar{t}_{k}(k)=2^{-2 k} p_{k, k}=\frac{1}{2}$. Since $\bar{t}_{k}$ is even, this leads to the equations

$$
\bar{t}_{k}(y)=\frac{1}{2} \prod_{j=0}^{k-1} \frac{\left(y^{2}-j^{2}\right)}{\left(k^{2}-j^{2}\right)}=\frac{1}{(2 k) !} \prod_{j=0}^{k-1}\left(y^{2}-j^{2}\right) .
$$

The theorem follows because $t_{S^{4 k-1}}\left(p_{k}^{*} E_{c}\right)=a_{k} \bar{t}_{k}(\sqrt{c}) \bmod \mathbb{Z}$. 


\section{ACKNOWLEDGEMENTS}

It is a pleasure to thank Wolfgang Ziller for raising questions which stimulated this paper and Nitu Kitchloo for his interest in the problem. We would also like to thank Matthias Kreck for helpful comments, Ian Hambleton for a tea-time discussion in which Theorem 1.12 was formulated, Uli Bunke for his continued interest in our work and Tibor Macko. Finally we would like to thank the referee for many detailed comments.

\section{REFERENCES}

1. J. F. Adams, On the groups $J(X)$. IV, Topology 5 (1966), 21-71. MR0198470 (33:6628)

2. D. W. Anderson, E. H. Brown and F. P. Peterson, The structure of the spin cobordism ring, Ann. of Math. (2) 86 (1967), 271-298. MR0219077(36:2160)

3. M. F. Atiyah, K-theory, Lecture notes by D. W. Anderson, W. A. Benjamin, Inc., New York-Amsterdam 1967. MR 0224083 (36:7130)

4. M. F. Atiyah and F. Hirzebruch, Riemann-Roch theorems for differentiable manifolds, Bull. Amer. Math. Soc. 65 (1959), 276-281. MR0110106 (22:989)

5. M. F. Atiyah, V. K. Patodi and I. M. Singer, Spectral asymmetry and Riemannian geometry. I, Math. Proc. Cambridge Philos. Soc. 77 (1975), 43-69; II Math. Proc. Cambridge Philos. Soc. 78 (1975), 405-432; III Math. Proc. Cambridge Philos. Soc. 79 (1976), 71-99. MR.0397797 (53:1655a) MR0397798(53:1655b) MR0397799(53:1655c)

6. M. F. Atiyah and I. M. Singer, The index of elliptic operators on compact manifolds, Bull. Amer. Math. Soc. 69 (1963), 422-433. MR0157392 (28:626)

7. J. M. Bismut and W. Zhang, An extension of a theorem by Cheeger and Müller, with an appendix by François Laudenbach, Astérisque No. 205 (1992), 235 pp. MR.1185803 (93j:58138)

8. __ Real embeddings and eta invariants, Math. Ann. 295 (1993), 661-684. MR.1214954 (94e:58131)

9. W. Browder, Surgery on simply-connected manifolds, Ergebnisse der Mathematik und ihrer Grenzgebiete, Band 65. Springer-Verlag, New York-Heidelberg 1972. MR 0358813 (50:11272)

10. U. Bunke, On the topological contents of eta invariants, preprint (2011), arXiv:1103.4217.

11. H. Cerf, Sur les difféomorphismes de la sphère de dimension trois $\left(\Gamma_{4}=0\right)$, Lecture Notes in Mathematics 53, Springer-Verlag, 1968. MR0229250 (37:4824)

12. D. Crowley, The classification of highly connected manifolds in dimensions 7 and 15, Ph.D. Thesis, Indiana University 2002, arXiv:math/0203253. MR2703475

13. D. Crowley and C. Escher, Classification of $S^{3}$-bundles over $S^{4}$, Diff. Geom. Appl. 18 (2003), 363-380. MR1975035(2004e:55020)

14. O. Dearricott, A 7-manifold with positive curvature, Duke Math. J. 158 (2011), 307-346. MR2805071

15. F. Deloup and G. Massuyeau, Quadratic functions and complex spin structures on threemanifolds, Topology 44 (2005), no. 3, 509-555. MR.2122215 (2005j:57033)

16. H. Donnelly, Spectral geometry and invariants from differential topology, Bull. London Math. Soc. 7 (1975), 147-150. MR0372929(51:9133)

17. J. Eells and N. Kuiper, An invariant for certain smooth manifolds, Annali di Math. 60 (1962), 93-110. MR0156356 (27:6280)

18. S. Feder and S. Gitler, Mappings of quaternionic projective spaces, Bol. Soc. Mat. Mexicana (2) 18 (1973), 33-37. MR0336740(49:1513)

19. S. Goette, Adiabatic limits of Seifert fibrations, Dedekind sums, and the diffeomorphism type of certain 7-manifolds, preprint (2011), arXiv:1108.5614.

20. D. L. Gonçalves and M. Spreafico, Quaternionic Line Bundles over Quaternionic Projective Spaces, Math. J. Okayama Univ. 48 (2006), 87-101. MR2291170(2008h:55013)

21. K. Grove, L. Verdiani and W. Ziller, An exotic $T_{1} S^{4}$ with positive curvature, Geom. Funct. Anal. 21 (2011), 499-524; arXiv:0809.2304. MR2810857

22. K. Grove, B. Wilking and W. Ziller, Positively Curved Cohomogeneity One Manifolds and 3-Sasakian Geometry, J. Diff. Geom. 78 (2008), 33-111; arXiv:math/0511464. MR.2406265 (2009m:53090) 
23. R. Hepworth, Generalized Kreck-Stolz invariants and the topology of certain 3-Sasakian 7manifolds, Ph.D. Thesis, University of Edinburgh, 2005.

24. M. Kreck, Surgery and duality, Ann. of Math. (2) 149 (1999), 707-754. MR.1709301 (2001a:57051)

25. M. Kreck and S. Stolz, A diffeomorphism classification of 7-dimensional homogeneous Einstein manifolds with $\mathrm{SU}(3) \times \mathrm{SU}(2) \times \mathrm{U}(1)$-symmetry, Ann. of Math. (2) 127 (1988), 373-388. MR932303 (89c:57042)

26. R. C Kirby and L. C. Siebenmann, Foundational essays on topological manifolds, smoothings, and triangulations, Ann. of Math. Stud. No. 88. Princeton University Press, Princeton, N.J.; University of Tokyo Press, Tokyo, 1977. MR0645390 (58:31082)

27. I. Madsen and J. Milgram, The classifying spaces for surgery and cobordism of manifolds, Ann. of Math. Stud., Princeton University Press, 1979. MR548575 (81b:57014)

28. I. Madsen, L. Taylor and B. Williams, Tangential homotopy equivalences, Comm. Math. Helv. 55 (1980), 445-484. MR593058 (82f:57015)

29. S. Sasao, On homotopy type of certain complexes, Topology 3 (1965), 97-102. MR0171281 $(30: 1512)$

30. N. Steenrod, The topology of fibre bundles, Princeton Mathematical Series No. 14., Princeton University Press, 1951. MR0039258(12:522b)

31. H. Toda, Composition methods in homotopy groups of spheres, Princeton University Press, 1962. MR0143217(26:777)

32. C. T. C. Wall, Classification problems in differential topology - VI, Topology 6 (1967), 273-296. MR0216510(35:7343)

33. Surgery on compact manifolds, Second edition. Edited and with a foreword by A. A. Ranicki. Mathematical Surveys and Monographs, 69, American Mathematical Society, Providence RI, 1999. MR.1687388 (2000a:57089)

34. _ Poincaré complexes: I, Ann. of Math. (2) 86 (1967), 213-245. MR0217791 (36:880)

35. G. W. Whitehead, Elements of homotopy theory, Graduate Texts in Mathematics, SpringerVerlag, 1978. MR.516508 (80b:55001)

36. D. Wilkens, Closed $(s-1)$-connected $(2 s+1)$-manifolds, $s=3,7$, Bull. London Math. Soc. 4 (1972), 27-31. MR0307258(46:6378)

Hausdorff Research Institute for Mathematics, Universität Bonn, Poppelsdorfer Allee 82, D-53115 Bonn, Germany

E-mail address: diarmuidc23@gmail.com

Mathematisches Institut, Universität Freiburg, Eckerstr. 1, 79104 Freiburg, GerMANY

E-mail address: sebastian.goette@math.uni-freiburg.de 